\title{
THEORY FOR THE DEVELOPMENT OF NEURON SELECTIVITY: ORIENTATION SPECIFICITY AND BINOCULAR INTERACTION IN VISUAL CORTEX ${ }^{1}$
}

\author{
ELIE L. BIENENSTOCK, ${ }^{2}$ LEON N COOPER, ${ }^{3}$ AND PAUL W. MUNRO \\ Center for Neural Science, Department of Physics, and Division of Applied Mathematics, Brown University, Providence, \\ Rhode Island 02912
}

Received June 5, 1981; Revised August 27, 1981; Accepted September 1, 1981

\begin{abstract}
The development of stimulus selectivity in the primary sensory cortex of higher vertebrates is considered in a general mathematical framework. A synaptic evolution scheme of a new kind is proposed in which incoming patterns rather than converging afferents compete. The change in the efficacy of a given synapse depends not only on instantaneous pre- and postsynaptic activities but also on a slowly varying time-averaged value of the postsynaptic activity. Assuming an appropriate nonlinear form for this dependence, development of selectivity is obtained under quite general conditions on the sensory environment. One does not require nonlinearity of the neuron's integrative power nor does one need to assume any particular form for intracortical circuitry. 'This is first illustrated in simple cases, e.g., when the environment consists of only two different stimuli presented alternately in a random manner. The following formal statement then holds: the state of the system converges with probability 1 to points of maximum selectivity in the state space. We next consider the problem of early development of orientation selectivity and binocular interaction in primary visual cortex. Giving the environment an appropriate form, we obtain orientation tuning curves and ocular dominance comparable to what is observed in normally reared adult cats or monkeys. Simulations with binocular input and various types of normal or altered environments show good agreement with the relevant experimental data. Experiments are suggested that could test our theory further.
\end{abstract}

It has been known for some time that sensory neurons at practically all levels display various forms of stimulus selectivity. They may respond preferentially to a tone of a given frequency, a light spot of a given color, a light bar of a certain length, retinal disparity, orientation, etc. We might, therefore, regard stimulus selectivity as a general property of sensory neurons and conjecture that the development of such selectivity obeys some general rule. Most attractive is the idea that some of the mechanisms by which selectivity develops in embryonic or early postnatal life are sufficiently general to allow a unifying theoretical treatment.

\footnotetext{
${ }^{1}$ This work was supported in part by United States Office of Naval Research Contract N00014-81-K-0136, the Fondation de France, and the Ittleson Foundation, Inc. We would like to express our appreciation to our colleagues at the Brown University Center for Neural Science for their interest and helpful advice. In particular, we thank Professor Stuart Geman for several useful discussions.

${ }^{2}$ Present address: I Laboratoire de statistique appliquée, Batiment 425, Université de Paris Sud, 91405 Orsay, France.

${ }^{3}$ To whom correspondence should be addressed at Center for Neural Science, Brown University, Providence, RI 02912.
}

In the present paper, we attempt to construct such a mathematical theory of the development of stimulus selectivity in cortex. It is based on (1) an elementary definition of a general index of selectivity and (2) stochastic differential equations proposed as a description of the evolution of the strengths of all synaptic junctions onto a given cortical neuron.

The ontogenetic development of the visual system, particularly of higher vertebrates, has been studied very extensively. Since the work of Hubel and Wiesel (1959, 1962), it has been known that almost all neurons in the primary visual cortex (area 17) of the normally reared adult cat are selective; they respond in a precise and sometimes highly tuned fashion to a variety of featuresin particular, to bars or edges of a given orientation and/ or those moving in a given direction through their receptive fields. Further work has shown that the response characteristics of these cortical cells strongly depend on the visual environment experienced by the animal during a critical period extending roughly from the 3rd to the 15th week of postnatal life (see, for example, Hubel and Wiesel, 1965; Blakemore and Van Sluyters, 1975; Buis- 
seret and Imbert, 1976; Frégnac and Imbert, 1978; Frégnac, 1979). Although these experiments show that visual experience plays a determining role in the development of selectivity, the precise nature of this role is still a matter of controversy.

Applying our general ideas to the development of orientation selectivity and binocular interaction in area 17 of the cat visual cortex, we obtain a theory based on a single mechanism of synaptic modification that accounts for the great variety of experimental results on monocular and binocular experience in normal and various altered visual environments. In addition, we obtain some new predictions.

It is known that various algorithms related to Hebb's principle of synaptic modification (Hebb, 1949) can account for the formation of associative and distributed memories (see, for example, Marr, 1969; Brindley, 1969; Anderson, 1970, 1972; Cooper, 1973; Kohonen, 1977). We therefore suggest that it may be the same fundamental mechanism, accessible to detailed experimental investigation in primary sensory areas of the nervous system, which is also responsible for some of the higher forms of central nervous system organization.

In sections I to III, our ideas are presented in general form, section IV is devoted to the development of orientation selectivity primarily in a normal visual environment, whereas in section $\mathrm{V}$, it is shown that our assumptions also account for normal or partial development of orientation selectivity and binocularity in various normal or altered visual environments.

\section{Preliminary Remarks and Definitions}

Notation. We simplify the description of the dynamics of a neuron by choosing as variables not the instantaneous incoming time sequence of spikes in each afferent fiber, the instantaneous membrane potential of the neuron, or the time sequence of outgoing spikes but rather the pre- and postsynaptic firing frequencies. These may be thought of as moving time averages of the actual instantaneous variables, ${ }^{4}$ where the length of the averaging interval is of the order of magnitude of the membrane time constant, $\tau$. Throughout this paper, these firing frequencies are used as instantaneous variables. This formal neuron is thus a device that performs spatial integration (it integrates the signals impinging all over the soma and dendrites) rather than spatiotemporal integration: the output at time $t$ is a function of the input and synaptic efficacies at $t$, independent of past history.

A synaptic efficacy $m_{j}$ characterizes the net effect of the presynaptic neuron $j$ on the postsynaptic neuron (in most of the paper, only one postsynaptic neuron is considered). This effect may be mediated through a complex system including perhaps several interneurons, some of which are excitatory and others inhibitory. The resulting "ideal synapse" (Nass and Cooper, 1975) thus may be of either sign, depending on whether the net effect is excitatory or inhibitory; it also may change sign during development.

\footnotetext{
${ }^{4}$ The precise form of the averaging integral (i.e., of the convolution kernel) is not essential. Exponential kernels $K(t)=\exp (-t / \tau)$ often are used in this context (see, e.g., Nass and Cooper, 1975; Uttley, 1976).
}

A further simplification is to assume that the integrative power of the neuron is a linear function, that is:

$$
c(t)=\Sigma_{j} m_{j}(t) d_{j}(t)
$$

where $c(t)$ is the output at time $t, m_{j}(t)$ is the efficacy of the $j$ th synapse at time $t, d_{j}(t)$ is the $j$ th component of the input at time $t$ (the firing frequency of the $j$ th presynaptic neuron), and $\Sigma_{j}$ denotes summation over $j$ (i.e., over all presynaptic neurons). We can then write:

$$
\begin{aligned}
m(t) & =\left(m_{1}(t), m_{2}(t), \ldots, m_{N}(t)\right) \\
d(t) & =\left(d_{1}(t), d_{2}(t), \ldots, d_{N}(t)\right) \\
c(t) & =m(t) \cdot d(t)
\end{aligned}
$$

$m(t)$ and $d(t)$ are real-valued vectors, of the same dimension, $N$ (i.e., the number of ideal synapses onto the neuron), and $c(t)$ is the inner product (or "dot product") of $m(t)$ and $d(t)$. The vector $m(t)$ (i.e., the array of synaptic efficacies at time $t$ ) is called the state of the neuron at time $t$. (Note that $c(t)$ as well as all components of $d(t)$ represent firing frequencies that are measured from the level of average spontaneous activity; thus, they might take negative as well as positive values; $m_{j}(t)$ is dimensionless.)

The precise form of the integrative power is not essential: our results remain unchanged if, for instance, $c(t)$ $=S(m(t) \cdot d(t))$, with $S$ being a positive-valued sigmoidshaped function (see Bienenstock, 1980). This is in contrast to other work (e.g., von der Malsburg, 1973) that does require nonlinear integrative power (see "Appendix B").

Selectivity. It is common usage to estimate the orientation selectivity of a single visual cortical neuron by measuring the half-width at half-height-or an equivalent quantity - of its orientation tuning curve. The selectivity then is measured with respect to a parameter of the stimulation, namely the orientation, which takes on values over an interval of $180^{\circ}$. In the present study, various kinds of inputs are considered, e.g., formal inputs with a parameter taking values on a finite set of points rather than a continuous interval. It will be useful then to have a convenient general index of selectivity, defined in all cases. We propose the following:

$$
\operatorname{Sel}_{d}(\mathscr{N})=1-\frac{\text { mean response of } \mathscr{N} \text { with respect to } d}{\text { maximum response of } \mathscr{N} \text { with respect to } d}
$$

With this definition, selectivity is estimated with respect to or in an environment for the neuron, that is, a random variable $\boldsymbol{d}$ that takes on values in the space of inputs to the neuron $\mathcal{N}$. The variable $\boldsymbol{d}$ represents a random input to the neuron; it is characterized by its probability distribution that may be discrete or continuous. (During normal development, the input to the neuron (or neuronal network) is presumably distributed uniformly over all orientations. In abnormal rearing conditions (e.g., dark reared), the input during development could be different from the input for measuring selectivity. How this should be translated in the formal space $R^{N}$ will be discussed in section IV.) This distribution defines an environment, mathematically a random variable $\boldsymbol{d}$. Selectivity is estimated (before or after develop- 
ment) with respect to this same environment. ${ }^{5}$ Obviously, $\operatorname{Sel}_{d}(\mathcal{N})$ always falls between 0 and 1 and the higher the selectivity of $\mathscr{N}$ in $\boldsymbol{d}$, the closer $\operatorname{Sel}_{\boldsymbol{d}}(\mathscr{N})$ is to 1.

When applied to the formal neuron in state $m$, definition 3 gives:

$$
\operatorname{Sel}_{\boldsymbol{d}}(m)=1-\frac{E[m \cdot \boldsymbol{d}]}{\operatorname{ess} \sup (m \cdot \boldsymbol{d})}
$$

where $\boldsymbol{d}$ is any $R^{N}$-valued random variable (the formal environment for the neuron). The symbol $E[\ldots]$ stands for "expected value of ..." (i.e., the mean value with respect to the distribution of $\boldsymbol{d}$ ) and "ess sup of ..." (essential supremum) is equivalent to "maximum of ..." in most common applications. This is illustrated in Figure 1.

\section{Modification of Cortical Synapses}

The various factors that influence synaptic modification may be divided broadly into two classes-those dependent on global and those dependent on local information. Global information in the form of chemical or electrical signaling presumably influences in the same way most (or all) modifiable junctions of a given type in a given area. Evidence for the existence of global factors that affect development may be found, for instance, in the work of Kasamatsu and Pettigrew (1976, 1979), Singer $(1979,1980)$, and Buisseret et al. (1978). On the other hand, local information available at each modifiable synapse can influence each junction in a different manner. In this paper, we are interested primarily in the effect of local information on the development of selectivity.

An early proposal as to how local information could affect synaptic modification was made by Hebb (1949). His, now classical, principle was suggested as a possible neurophysiological basis for operant conditioning: "when an axon of cell A is near enough to excite a cell B and repeatedly or persistently takes part in firing it, some growth process or metabolic change takes place in one or both cells such that A's efficiency, as one of the cells firing B, is increased." Thus, the increase of the synaptic strength connecting $\mathrm{A}$ to $\mathrm{B}$ is dependent upon the correlated firing of A and B. Such a correlation principle has inspired the work of many theoreticians on various topics related to learning, associative memory, pattern recognition, the organization of neural mappings (retinotopic projections), and the development of selectivity of cortical neurons.

It is fairly clear that, in order to actually use Hebb's principle, one must state conditions for synaptic decrease as specific as those for synaptic increase: if synapses are allowed only to increase, all synapses will eventually saturate; no information will be stored and no selectivity

\footnotetext{
${ }^{5}$ The mathematical concept that is needed in order to represent the environment, $\boldsymbol{d}$, during the development period is that of a stationary stochastic process, $\boldsymbol{d}(t)$, that is (roughly), a time-dependent random variable whose distribution is invariant in time. For example, $\boldsymbol{d}$ could represent an elongated bar in the receptive field of the neuron, rotating in some random manner around its center. At each instant, the probability of finding the bar in any given orientation is the same as at any other: the distribution of $\boldsymbol{d}(t)$ is time invariant, uniform over the interval $\left(0,180^{\circ}\right)$.
}

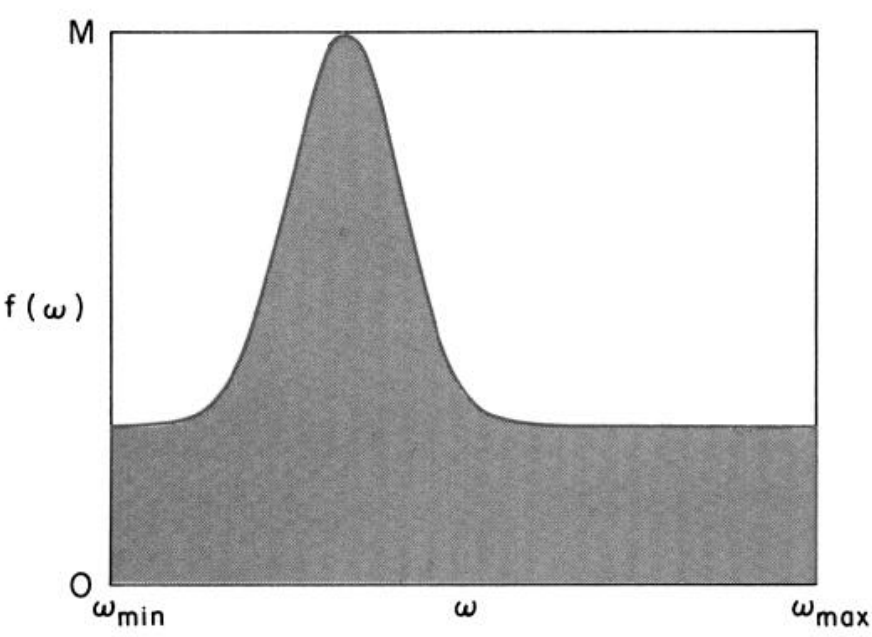

Figure 1. Computing the selectivity with respect to an environment uniformly distributed between $\omega_{\min }$ and $\omega_{\max }$. The abscissa displays a parameter of the stimulus (e.g., orientation $\left.\left(\omega_{\max }-\omega_{\min }=180^{\circ}\right)\right)$ and on the ordinate, the neuron's response 0 is the level of the average spontaneous activity; $M$ is the maximum response. The selectivity of the neuron then is given by

$$
\begin{aligned}
& \operatorname{Sel}_{d}(\mathscr{N})=1-\frac{1}{M\left(\omega_{\max }-\omega_{\min }\right)} \int_{\omega_{\min }}^{\omega_{\max }} f(\omega) d \omega \\
&=\frac{\text { light area }}{\text { total box area }}
\end{aligned}
$$

This is a simple measure of the breadth of the peak: curves of same selectivity have approximately the same half-width at half-height. (Think, for instance, of triangularly shaped tuning curves.) Typical values for orientation selectivity of adult cortical cells vary between 0.7 and 0.85 ("specific" cells). Selectivity of broadly tuned but still unimodal cells (e.g., those termed "immature" by Buisseret and Imbert (1976) and Frégnac and Imbert (1978)) lies between 0.5 and 0.7 . Obviously, 0 is the selectivity of an absolutely flat curve, whereas 1 is the selectivity of a Dirac $\delta$ function.

will develop (see, for example, Sejnowski, 1977a, b). What is required is thus a complementary statement to Hebb's principle giving conditions for synaptic decrease. ${ }^{6}$

Such statements usually have resulted in a form of synaptic competition. Consider, for example, one that was proposed by Stent (1973): "when the presynaptic axon of cell A repeatedly and persistently fails to excite the postsynaptic cell $B$ while cell $B$ is firing under the influence of other presynaptic axons, metabolic changes take place in one or both cells such that A's efficiency, as one of the cells firing B, is decreased." According to Stent's principle, the increase of the strength of certain synapses onto neuron B is accompanied by simultaneous decrease of the strength of other synapses onto the same

\footnotetext{
${ }^{6}$ Nonspecific conditions for synaptic decrease, such as uniform exponential decay, are clearly insufficient too: in Nass and Cooper (1975) for instance, no selectivity is achieved without lateral intracortical inhibition. Other models (von der Malsburg, 1973; Perez et al., 1975) use a normalization rule in conjunction with a hebbian scheme for synaptic increase, which actually results in decrease as well as increase. This normalization rule is discussed in "Appendix B."
} 
neuron $\mathrm{B}$. There thus occurs a spatial competition between convergent afferents. A competition mechanism of this kind provides a qualitative explanation of some experimental results on cortical development (e.g., monocularly deprived animals (Stent, 1973)) as well as some aspects of certain more complex deprivation paradigms such as those recently reported by Rauschecker and Singer (1981).

In the present work, we present a mechanism of synaptic modification that results in a temporal competition between input patterns rather than a spatial competition between different synapses. With this mechanism, whether synaptic strength increases or decreases depends upon the magnitude of the postsynaptic response as compared with a variable modification threshold. We show that this can account quantitatively in a more powerful way for increases and decreases in selectivity as well as for a great variety of other experimental results in diverse rearing conditions.

We propose that the change of the $j$ th synapse's strength at the time $t$ obeys the following rule:

$$
\dot{\boldsymbol{m}}_{j}(t)=\phi(c(t)) \boldsymbol{d}_{j}(t)-\epsilon \boldsymbol{m}_{j}(t)
$$

where $\phi(c)$ is a scalar function of the postsynaptic activity, $c(t)$, that changes sign at a value, $\theta_{M}$, of the output called the modification threshold:

$$
\phi(c)<0 \text { for } c<\theta_{M} ; \quad \phi(c)>0 \text { for } c>\theta_{M}
$$

The term, $-\epsilon m(t)$, produces a uniform decay of all junctions; this, in most cases, does not affect the behavior of the system if $\epsilon$ is small enough. However, as will be seen later, it is important in some situations. Other than this uniform decay, the vector $m$ is driven in the direction of the input $d$ if the output is large (above $\theta_{M}$ ) or opposite to the direction of the input if the output is small (below $\left.\theta_{M}\right)$. As required by Hebb's principle, when $d_{j}>0$ and $c$ is large enough, $\boldsymbol{m}_{j}$ increases. However, when $\boldsymbol{d}_{j}>0$ and $\boldsymbol{c}$ is not large enough, $\boldsymbol{m}_{j}$ decreases. We may regard this as a form of temporal competition between incoming patterns.

The idea of such a modification scheme was introduced by Cooper et al. (1979). Their use of a constant threshold $\theta_{M}$, however, resulted in a certain lack of robustness of the system: the response to all patterns could slip below $\theta_{M}$ and then decrease to zero. In the absence of lateral inhibition between neurons, the response might increase to more than one pattern, leading to stable states with a maximal response to more than one pattern.

In this paper, we will see that making an appropriate choice for $\theta_{M}(t)$ allows correct functioning under quite general conditions and provides remarkable noise tolerance properties.

In our threshold modification scherne, the change of the $j$ th synapse's strength is written as a product of two terms, the presynaptic activity, $\boldsymbol{d}_{j}(t)$, and a function, $\phi(c(t), \bar{c}(t))$, of the postsynaptic variables, the output, $\boldsymbol{c}(t)$, and the average output, $\overline{\boldsymbol{c}}(t)$. Making use of $\overline{\boldsymbol{c}}(t)$ in the evolutive power of the neuron is a new and essential feature of this work. It is necessary in order to allow both boundedness of the state and efficient threshold modification.

Neglecting the uniform decay term, for the moment $(\epsilon=0)$, in vector notation, we have

$$
\dot{\boldsymbol{m}}(t)=\phi(\boldsymbol{c}(t), \overline{\boldsymbol{c}}(t)) d(t)
$$

This, together with equation 2 , yields:

$$
\dot{\boldsymbol{m}}(t)=\phi(\boldsymbol{m}(t) \cdot \boldsymbol{d}(t), \boldsymbol{m}(t) \cdot \bar{d}) \boldsymbol{d}(t)
$$

The crucial point in the choice of the function $\phi(c, \bar{c})$ is the determination of the threshold $\theta_{M}(t)$ (i.e., the value of $c$ at which $\phi(c, \bar{c})$ changes sign). A candidate for $\theta_{M}(t)$ is the average value of the postsynaptic firing rate, $\bar{c}(t)$. The time average is meant to be taken over a period $T$ preceding $t$ much longer than the membrane time constant $\tau$ so that $\overline{\boldsymbol{c}}(t)$ evolves on a much slower time scale than $c(t)$. This usually can be approximated ${ }^{7}$ by averaging over the distribution of inputs for a given state $\boldsymbol{m}(t)$

$$
\overline{\boldsymbol{c}}(t)=\boldsymbol{m}(t) \cdot \bar{d}
$$

This results in an essential feature, the instability of low selectivity points. (This can be most easily seen at 0 selectivity equilibrium points, where, with any perturbation, the state is driven away from this equilibrium, whatever the input.)

Therefore, if stable equilibrium points exist in the state space, they are of high selectivity. However, do such points exist at all? The answer is generally yes provided that the state is bounded from the origin and from infinity. These conditions, instability of low selectivity equilibria as well as boundedness, are fulfilled by a single function $\phi(c, \bar{c})$ if we define $\theta_{M}(t)$ to be a nonlinear function of $c(t)$ (for example, a power with an exponent larger than 1). The final requirement on $\phi(c, \bar{c})$ thus reads:

$$
\begin{aligned}
& \operatorname{sign} \phi(c, \bar{c})=\operatorname{sign}\left(c-\left(\frac{\bar{c}}{c_{0}}\right)^{D} \bar{c}\right) \quad \text { for } c>0 \\
& \phi(0, \bar{c})=0 \text { for all } \bar{c}
\end{aligned}
$$

where $c_{0}$ and $p$ are two fixed positive constants. ${ }^{8}$ The

\footnotetext{
${ }^{7}$ Replacing the line average by an average over the distribution of $\boldsymbol{d}$ is allowed provided that $(1)$ the process $\boldsymbol{d}(t)$ is stationary, (2) the interval, $T$, of time integration is short with respect to the process of synaptic evolution (i.e., $\boldsymbol{m}(t)$ changes very little during an interval of length $T$ ), (3) $T$ is long compared to the mixing rate of the process $d$ (i.e., during a period of length $T$, the relative time spent by the process $\boldsymbol{d}(t)$ at any point $d$ in the input space is nearly proportional to the weight of the distribution of $d$ at $d$ ). Now, synaptic modification of the type involved in changes of selectivity is probably a slow process, requiring minutes or hours (if not days) to be significant, whereas elementary sensory patterns (e.g., oriented stimuli in the receptive field of a given cortical neuron) are normally all experienced in an interval of the order of $1 \mathrm{~min}$ or less. Thus, we are able to choose $T$ so that a good estimate of $\bar{c}(t)$ can be available to the neuron. In some experimental situations in which the environment is altered, there are subtle dependences of the sequence by which the final state is reached depending on how rapidly $\bar{c}$ adjusts to the changed environment.

${ }^{8}$ The sign of $\phi(c, \bar{c})$ for $c<0$ is not crucial since $c$ is essentially a positive quantity: cortical cells in general have low spontaneous activity and, at any rate, are rarely inhibited much below their spontaneous activity level. For the sake of mathematical completeness, one may, however, wish to define $\phi(c, \bar{c})$ for negative $c ; \phi(c, \bar{c})>0$ is then the most convenient for it allows us to state theorems 1 to 3 below under the most general initial conditions. In addition, the form of $\phi$ for $c<0$ can affect calculations such as those of "Appendix C."
} 


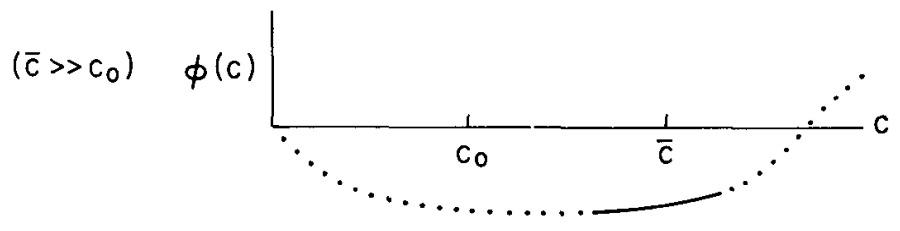

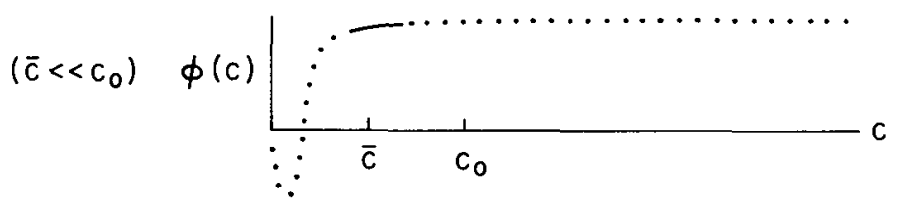

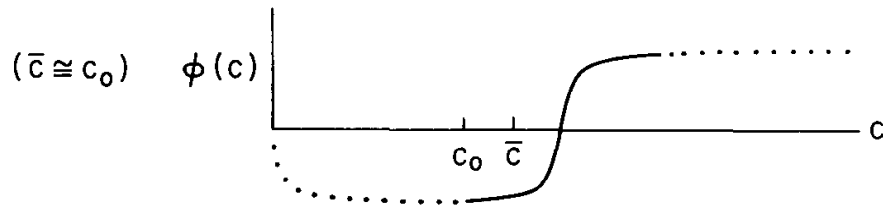

Figure 2. A function satisfying condition 7. The three diagrams show the behavior of $\phi(c, \bar{c})$ as a function of $c$ for three different constant values of $\bar{c}$. In each diagram, the solid part of the curve represents $\phi(c, \bar{c})$ in the vicinity of $\bar{c}$, which of course is the relevant part of this function. In the upper diagram $\left(\bar{c} \gg c_{0}\right)$, although $\phi(c, \vec{c})$ is not negative for all $c$ values as was formally required (see text), the probability that $\phi(c, \bar{c})$ $>0$ is small and gets even smaller as $\bar{c}$ increases. The important point in the definition of $\phi$ is the nonlinearity of $\theta_{M}(\bar{c})$ which makes it increase or decrease faster than $\bar{c}$, while $\theta_{M}(\bar{c})$ is of the same order as $\bar{c}$, if $\bar{c}$ itself is of the same order as $c_{0}$.

threshold $\theta_{M}(\bar{c})=\left(\bar{c} / c_{0}\right)^{p} \bar{c}$ thus serves two purposes: allowing threshold modification when $\bar{c} \simeq c_{0}$ as well as driving the state from regions such that $\bar{c} \ll c_{0}$ or $\bar{c} \gg c_{0}$. Fquation 7 is illustrated in Figure 2.

The process of synaptic growth, starting near zero to eventually end in a stable selective state, may be described as follows. Initially, $\bar{c} \ll c_{0}$; hence, $\phi(c, \bar{c})>0$ for all inputs in the environment: the responses to all inputs grow. With this growth, $\bar{c}$ increases, thus increasing $\theta_{M}$. Now some inputs result in postsynaptic responses that exceed $\theta_{M}$, while others-those whose direction is far away from (close to orthogonal to) the favored inputsgive a response less than $\theta_{M}$. The response to the former continues to grow, while the response to the latter decays. This results in a form of competition between incoming patterns rather than competition between synapses. The response to unfavored patterns decays until it reaches zero, where it stabilizes for $\phi(0, \bar{c})=0$ for any $\bar{c}$ (equation 7 ). The response to favored patterns grows until the mean response $\bar{c}$ is high enough, and the state stabilizes. This occurs in spite of the fact that many complicated geometrical relationships may exist between different patterns (i.e., that they are not orthogonal since different patterns may and certainly do share common synapses).

Any function, $\phi$, that satisfies equation 7 will give the results that we describe below. The precise form of this function (e.g., the numerical values of $p$ and $c_{0}$ ) will affect the detailed behavior of the system, such as rate of convergence, the height of the maximum response for a selective cell, etc., and would have to be determined by experiment.

\section{Mathematical Results}

The behavior of system 6 depends critically on the environment, that is, on the distribution of the stationary stochastic process, $\boldsymbol{d}$. Two classes of distributions may be considered-discrete distributions and continuous distributions. Discrete distributions include $K$ possible inputs $d^{1}, \ldots, d^{K}$. These will generally be assumed to occur with the same probability $1 / K$. The process $d$ is then a jump process which randomly assumes new values at each time increment. The vector $\boldsymbol{m}$ is (roughly) a Markov process. In the present work, the only continuous distribution that will be considered is a uniform distribution $d$ over a closed one-parameter curve in the input space $R^{N}$ (section IV).

Although the principles underlying the convergence to selective states are intuitively fairly simple (see the preceding section), mathematical analysis of the system is not entirely straightforward, even for the simplest $\boldsymbol{d}$. Mathematical results, obtained only for certain discrete distributions, are of two types: (1) equilibrium points are locally stable if and only if they are of the highest available selectivity with respect to the given distribution of $\boldsymbol{d}$ and (2) given any initial value of $\boldsymbol{m}$ in the state space, the probability that $\boldsymbol{m}(t)$ converges to one of the maximum selectivity fixed points as $t$ goes to infinity is 1. Results of the second type are much stronger and require a tedious geometrical analysis. Results are stated here in a somewhat simplified form (obvious requirements of a very mathematical character are omitted). For exact statements and proofs, the reader is referred to Bienenstock (1980).

We first study the simple case where $\boldsymbol{d}$ takes on values on only two possible input vectors, $d^{1}$ and $d^{2}$, that occur with the same probability:

$$
P\left[d=d^{1}\right]=P\left[d=d^{2}\right]=1 / 2
$$

Whatever the actual dimension $N$ of the system, it reduces to two dimensions. (Any component of $\boldsymbol{m}$ outside of the linear subspace spanned by $d^{1}$ and $d^{2}$ will eventually decay to zero duc to the uniform decay term.)

It follows immediately from the definition that the maximum value of $\operatorname{Sel}_{d}(m)$ in the state space is $1 / 2$. It is reached for states $m$ which give a null response when $d^{1}$ comes in (i.e., are orthogonal to $d^{1}$ ) but a positive response for $d^{2}$-or vice versa. Minimum selectivity, namely zero, is obtained for states $m$ such that $m \cdot d^{1}=$ $m \cdot d^{2}$. Equilibrium states of both kinds indeed exist.

Lemma 1. Let $d^{1}$ and $d^{2}$ be linearly independent and $d$ satisfy $P\left[\boldsymbol{d}=d^{1}\right]=P\left[\boldsymbol{d}=d^{2}\right]=1 / 2$. Then for any value of $\phi$ satisfying equation 7, equation 6 admits exactly four fixed points, $m^{0}, m^{1}$, $m^{2}$, and $m^{1,2}$ with: $\operatorname{Sel}_{d}\left(m^{0}\right)=\operatorname{Sel}_{d}\left(m^{1,2}\right)=0$ and $\operatorname{Sel}_{d}\left(m^{1}\right)=$ $\operatorname{Sel}_{d}\left(m^{2}\right)=1 / 2$. (Here the superscripts indicate which of the $d^{i}$ are not orthogonal to $m$. ( $m^{0}$ is the origin.) Thus, for instance, $m^{1} \cdot d^{1}>0, m^{1} \cdot d^{2}=0$.)

The behavior of equation 6 depends on the geometry of the inputs, in the present case, on $\cos \left(d^{1}, d^{2}\right)$. The crucial assumption needed here is that $\cos \left(d^{1}, d^{2}\right) \geqslant 0$. This is a reasonable assumption which is obviously satisfied if all components of the inputs are positive, as is assumed in some models (von der Malsburg, 1973; Perez et al., 1975). We then may state the following.

Theorem 1. Assume that, in addition to the conditions of 
lemma $1, \cos \left(d^{1}, d^{2}\right) \geqslant 0$. Then $m^{0}$ and $m^{1,2}$ are unstable, $m^{1}$ and $m^{2}$ are stable, and whatever its initial value, the state of the system converges almost surely (i.e., with probability 1) either to $m^{1}$ or to $m^{2}$.

Theorem 1 is the basic result in the two-dimensional setting: it characterizes evolution schemes based on competition between patterns and states that the state eventually reaches maximal selectivity even when the two input vectors are very close to one another. Obviously this requires that some of the synaptic strengths be negative since the neuron has linear integrative power. Inhibitory connections are thus necessary to obtain selectivity (see also section IV below). Some selectivity is also realizable with no inhibitory connections-not even "intracortical" ones-if the integrative power is appropriately nonlinear. However, whatever the nonlinearity of the integrative power, theorem 1 could not hold for evolution equations based on competition between converging afferents (see "Appendix B").

In theorem 1, we have a discrete sensory environment which consists of exactly two different stimuli-a situation, although simple mathematically, not often encountered in nature. It may, however, very well correspond to a visual environment restricted to only horizontally and vertically oriented contours present with equal probability. Theorem 1 then predicts that cortical cells will develop a selective response to one of the two orientations, with no preference for either (other than what may result from initial connectivity). Thus, on a large sample of cortical cells, one should expect as many cells tuned to the horizontal orientation as to the vertical one. (So far, no assumption is made on intracortical circuitry. See "Appendix D.")

The proof of theorem 1 is based on the existence of trap regions around each of the selective fixed points.

Theorem 2. Under the same conditions as in theorem 1 , there exists around $m^{1}\left(m^{2}\right)$ a region $F^{1}\left(F^{2}\right)$ such that, once the state enters $F^{1}\left(F^{2}\right)$, it converges almost surely to $m^{1}\left(m^{2}\right)$.

The meaning of theorem 2 is the following: once $\boldsymbol{m}(t)$ has reached a certain selectivity, it cannot "switch" to another selective region. Applied to cortical cells in a patterned visual environment, this means that, once they become sufficiently committed to certain orientations, they will remain committed to those orientations (provided that the visual environment does not change), becoming more selective as they stabilize to some maximal selectivity. Theorems 1 and 2 are illustrated in Figure 3 .

It is worth mentioning that, when $\cos \left(d^{1}, d^{2}\right)<0$, the situation is much more complicated: trap regions do not necessarily exist and periodic asymptotic behavior (i.e., limit cycles) may occur, bifurcating from the stable fixed points when $\cos \left(d^{1}, d^{2}\right)$ becomes too negative (see Bienenstock, 1980).

We now turn to the case where $\boldsymbol{d}$ takes on $K$ values. The following is easily obtained.

Lemma 2. Let $d^{1}, d^{2}, \ldots, d^{K}$ be linearly independent and $\boldsymbol{d}$ satisfy $P\left[\boldsymbol{d}=d^{1}\right]=\ldots=P\left[\boldsymbol{d}=d^{K}\right]=1 / K$. Then, for any function $\phi$ satisfying equation 7 , equation 6 admits exactly $2^{K}$ fixed points with selectivities $0,1 / K, 2 / K, \ldots,(K-1) / K$. There are $K$ fixed points $m^{1}, \ldots, m^{K}$ of selectivity $(K-1) / K$.

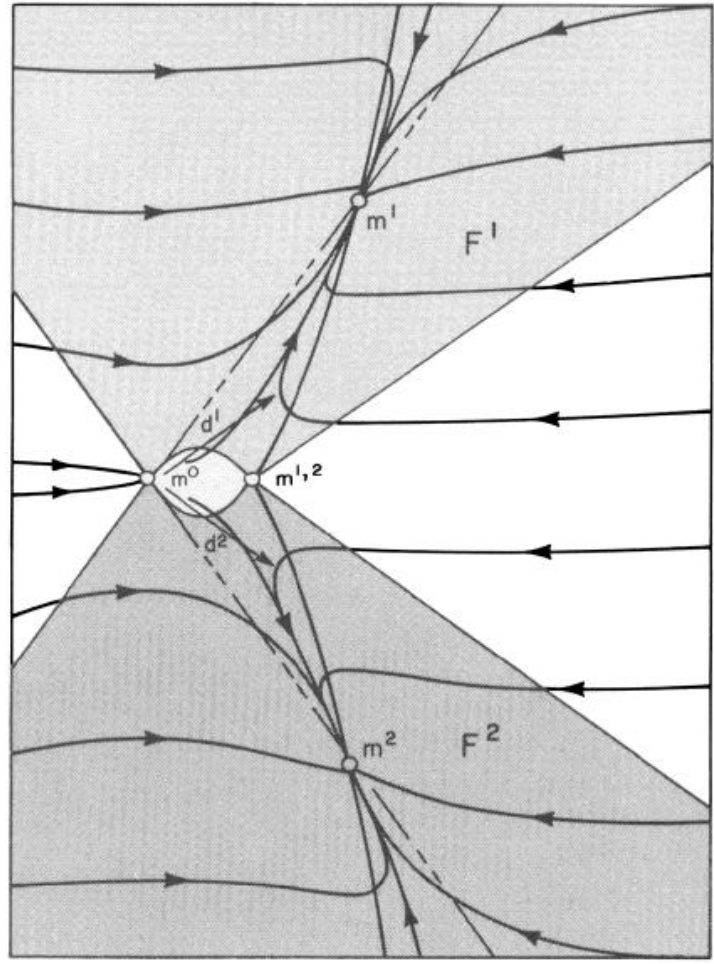

Figure 3. The phase portrait of equation 6 in an environment consisting of two inputs, $d^{1}$ and $d^{2}$ (theorems 1 and 2). The diagram shows the trajectories of the state of the system, starting from different initial points. This is a computer simulation performed with one given function $\phi$ satisfying condition 7. Using a different function may slightly change the shape of the trajectories without any essential change in the behavior. The unstable fixed points are $m^{1,2}$ and $m^{0}$; the stable ones are $m^{1}$ and $m^{2}$. The system is a stochastic one, which means that the trajectories depend, in fact, on the precise sequence of inputs. As long as the state is in the unshaded region, it is not yet known whether it will eventually be attracted to $m^{1}$ or $m^{2}$. This is determined as the state enters one of the trap (shaded) regions, $F^{1}$ or $F^{2}$. The trajectories shown here are deterministic ones, obtained by alternating $\boldsymbol{d}$ regularly between $d^{1}$ and $d^{2}$. They are, in fact, the averaged trajectories of the state and are much more regular and smooth than the actual stochastic ones.

Obviously, $(K-1) / K$ is also the maximum possible selectivity with respect to $d$. It means a positive response for one and only one of the inputs. The situation is now much more complicated than what it was with only two inputs: it is not obvious whether, in all cases, the assumption that all of the cosines between inputs are positive is sufficient to yield stability of the maximum selectivity fixed points. However, we may state the following.

Theorem 3. Assume, in addition to the conditions of lemma 2 , that $d^{1}, \ldots, d^{K}$ are all mutually orthogonal or close to orthogonal. Then the $K$ fixed points of maximum selectivity are stable, and whatever its initial value, the state of the system converges almost surely to one of them.

The proof of theorem 3 also involves trap regions around the $K$ maximally selective fixed points, and the analog of theorem 2 is true here.

Although the general case has not yet been solved analytically, as will be seen in the next section, computer 
simulations suggest that, for a fairly broad range of environments, if $d^{i} \cdot d^{j} \geqslant 0$, even if $d^{1}, \ldots, d^{K}$ are far from being mutually orthogonal, the $K$ fixed points of maximum selectivity are stable.

Simulations suggest further (see, for instance, Fig. 4b) that, even if the $d^{1}, \ldots, d^{K}$ are not linearly independent and are very far from being mutually orthogonal, the asymptotic selectivity is close to its maximum value with respect to $\boldsymbol{d}$.

\section{Orientation Selectivity and Binocular Interaction in Visual Cortex}

We now apply what has been done to a concrete example, orientation selectivity and binocular interaction in the primary visual cortex. The ordinary development of these properties in mammals depends to a large extent on normal functioning of the visual system (i.e., normal visual experience) during the first few weeks or months of postnatal life. This has been demonstrated many times by various experiments, based mainly on the paradigm of rearing the animal in a restricted sensory environment. In the next two sections, it is shown how equations 4 to 7 account for both normal development as well as development in restricted visual environments.

Consider first a classical test environment used to construct the tuning curve of cortical neurons. 'This environment consists of an elongated light bar successively presented or moved in all orientations-preferably in a random sequence-in the neuron's receptive field. Thus, all of the parameters of the stimulus are constant except one, the orientation, which is distributed uniformly on a circularly symmetric closed path. We assume that the retinocortical pathway maps this family of stimuli to the cortical neuron's space of inputs in such a way as to preserve the circular symmetry (as defined below). Thus, the typical theoretical environment that will be used for constructing the formal neuron's tuning curve is a random variable $\boldsymbol{d}$ uniformly distributed on a circularly symmetric closed one-parameter family of points in the space $R^{N}$. The parameter coding orientation in the receptive field is, in principle, continuous. However, for the purpose of numerical simulations, the distribution is made discrete. Thus, $\boldsymbol{d}$ takes on values on the points $d^{1}$, $\ldots, d^{K}$.

The requirement of circular symmetry is expressed mathematically as follows: the matrix of inner products of the vectors $d^{1}, \ldots, d^{K}$ is circular (i.e., each row is obtained from its nearest upper neighbor by shifting it one column to the right) and the rows of the matrix are unimodal. A random variable, $\boldsymbol{d}$, uniformly distributed on such a set of points will be, hereafter, called a circular environment. Such a $\boldsymbol{d}$ may be roughly characterized by three parameters: $N, K$, and a measure of the mutual geometrical closeness of the $d^{i}$ vectors, for instance, $\min \cos \left(d^{1}, d^{i}\right)$.

Now we are faced with the difficult problem of specifying the stationary stochastic process that represents the time sequence of inputs to the neuron during development. In a first analysis, there is no choice but to oversimplify the problem by giving the stochastic process exactly the same distribution as the circular $\boldsymbol{d}$ defined above. In doing so, we assume that development of orientation selectivity is to a large extent independent of other parameters of the stimulus (e.g., contrast, shape, position in the receptive field, retinal disparity for binocular neurons, etc.). The elementary stimulus for a cortical neuron is a rectilinear contrast edge or bar. Any additional pattern present at the same time in the receptive field is regarded as random noise. (A discussion of this point is given in Cooper et al. (1979).)

\section{IVa. Normal Monocular Input}

The behavior of a monocular system in circular environments is investigated by numerically simulating equation 6 with a variety of circular environments, $\boldsymbol{d}$, and functions $\phi$ satisfying equation 7 . In the simulations presented here, the dimension of the input and state space is generally $N=37$; the number $K$ of input vectors varies from 12 to 60 . (Various kinds of functions $\phi$ were used: some were stepwise constant; others were smooth, bounded, or unbounded.) One may reasonably expect the system's behavior to be fairly independent of $N$ and $K$ if these are high enough. However, the geometry of $\boldsymbol{d}$ may be determining: if the inputs, $d^{i}$, are closely packed together in the state space (i.e., if min $\cos \left(d^{1}, d^{i}\right)$ is close to 1 , convergence to selective states may presumably be difficult to achieve or even impossible.

Simulations show the following behavior:

1. The state converges rapidly to a fixed point or attractor.

2. Various such attractors exist. For a given $\boldsymbol{d}$ and $\phi$, they all have the same selectivity, which is close to its maximum value in $\boldsymbol{d}$.

3. The asymptotic tuning curve is always unimodal. Thus, one may talk of the preferred orientation of an attractor.

4. There exists an attractor for each possible orientation.

5. If there is no initial preference, all orientations have equal probability of attracting the state. (Which one will become favored depends on the exact sequence of inputs.) This does not hold for environments which are not perfectly circular, at least for a single neuron system such as the one studied here.

In Figure 4, $a$ and $b$ show, respectively, the progressive buildup of selectivity and the tuning curve when the state has virtually stabilized.

In summary then, the system behaves in circular environments exactly as we might have expected from the results of the preceding section. However, one should note one important difference: the maximum selectivity for a continuous environment cannot be calculated as simply as it was before. It is only when $\boldsymbol{d}$ is distributed uniformly on $K$ linearly independent vectors that we know that max $\operatorname{Sel}_{d}(m)=(K-1) / K$ (lemma 2). Theorem 3 indicates that, if, in addition, the vectors are nearly orthogonal to one another, this selectivity is indeed asymptotically reached. We could not prove that this is also true when the vectors are arranged circularly but are not mutually orthogonal. However, it could not be disproved by any numerical simulation; therefore, we conjecture that this is indeed true. (Reasonable selectivity is attained even in most unfavorable environments. As an 

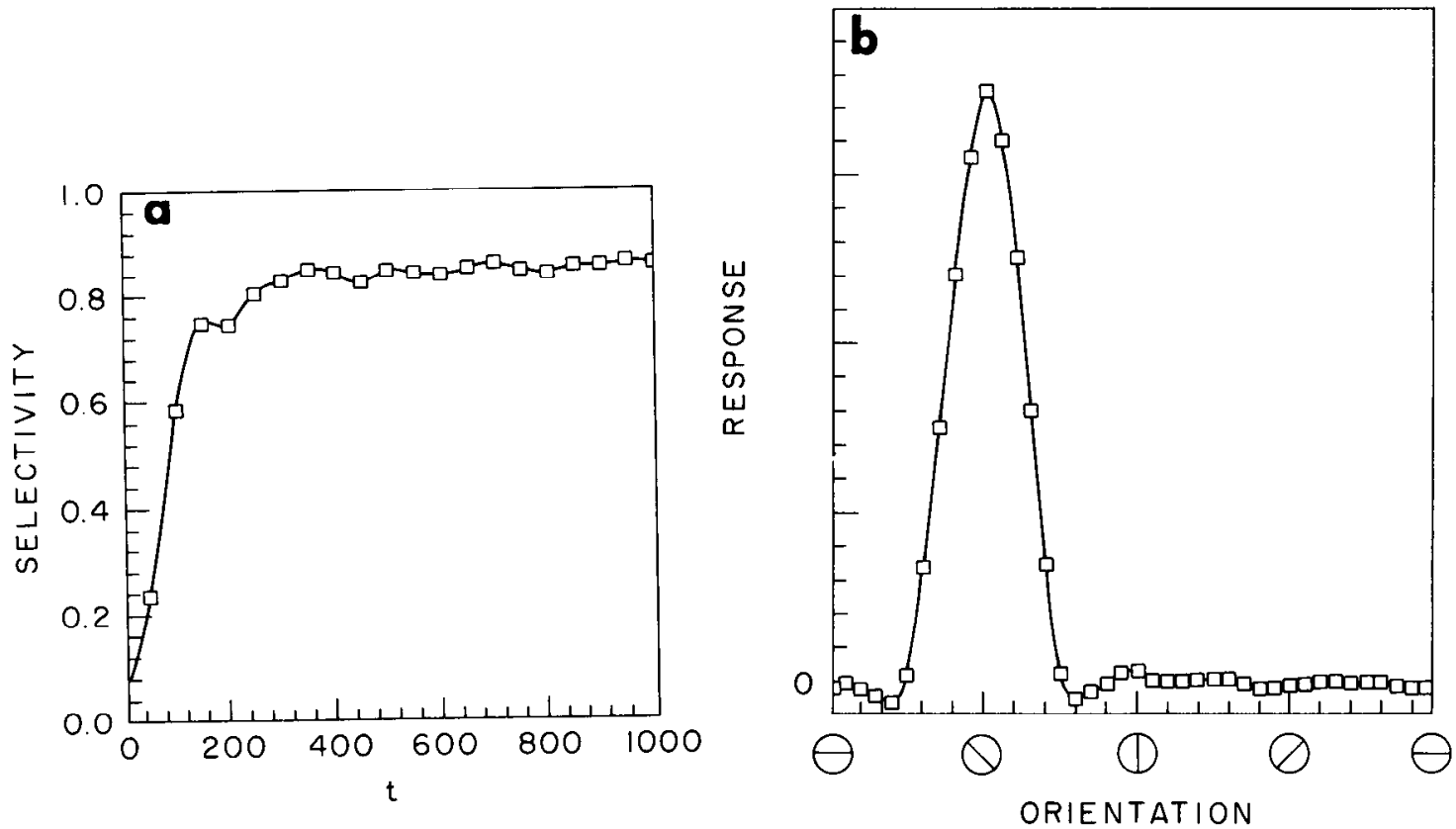

Figure 4. The evolution of a synaptic system in a circular environment. Here, $K=40$ and $N=37$ so that the vectors are linearly dependent. The value of the maximum selectivity with respect to $d$ is therefore not precisely calculable. The asymptotic selectivity is approximately 0.9 , perhaps the maximum selectivity. $a$ demonstrates the progressive buildup of the selectivity in a circular environment $d$, while $b$ shows the resulting tuning curve at $t=1000$.

example, in a circular $\boldsymbol{d}$ such that all cosines fall between 0.94 and 1 , a selectivity of 0.68 was reached after 12,000 iterations.) Notice that, in the present context, this question is only of theoretical interest, since naturally occurring environments are continuous rather than discrete. The behavior of our system in such an environment is very well approximated by a discrete circular $\boldsymbol{d}$, provided that $K$ is large enough. $K$ is then presumably larger than $N$, the $K$ inputs are linearly dependent, and we have no explicit formula for max $\operatorname{Sel}_{d}(m)$.

The system thus functions well in a large class of environments. It should be stressed that the numerical value of the parameters that appear explicitly in the evolution equation, namely, $c_{0}$ and the exponent $p$, are not at all critical. Simulations performed with a constant $\boldsymbol{d}$, with $p$ being varied from 0.01 to 10 , yield the same asymptotic limit for the selectivity; the height of the asymptotic tuning curve (i.e., $\max (m \cdot d)$ ) is, however, highly dependent on $p$. This invariance property validates in a sense the definition of $\operatorname{Sel}_{d}(x)$.

Inhibitory synapses are essential here exactly as they are in the two-dimensional case. One way to show this is to substitute 0 for all negative components in the state once it has become selective. This typically results in a drastic drop of selectivity (e.g., from 0.81 to 0.55 ) although a slight preference generally remains for the original orientation. This may be related to the experimental finding that local pharmacological deactivation of inhibitory connections strongly impairs orientation selectivity by rendering all orientations effective in triggering the cell's response (Sillito, 1975).

Finally, it should be mentioned that the system displays a good noise tolerance, particularly when the state has already reached a selective region. The system then resists presynaptic additive noise with a signal-to-noise ratio of the order of 1 and postsynaptic noise with a signal-to-noise ratio as small as $1 / 4$.

\section{IVb. Restricted Monocular Input}

To discuss this situation, we now must include the exponential decay term, $-\epsilon_{m}(t)$, previously neglected (equation 4). It is clear that the results stated above will be preserved if $\epsilon$ is sufficiently smaller than the average of $|\phi(c, \bar{c})|$ (i.e., competition mechanisms are faster than decay). However, exponential decay does become crucial in some situations. One of these is the response of the cell to patterns that were not represented in the environment during development.

Consider, for instance, an environment consisting of a single stimulus $d^{1}$. Il is then easily shown that system 6 with condition 7 admits one attractor $m^{1}$ that satisfies $m^{1} \cdot d^{1} \simeq c_{0}$ for small $\epsilon\left(m^{1} \cdot d^{1}=c_{0}\right.$ for $\left.\epsilon=0\right)$. Obviously, for $\epsilon>0, m^{1}$ will satisfy $m^{1} \cdot d=0$ for any $d$ orthogonal to $d^{1}$. However, the response to a pattern $d$ not orthogonal to $d^{1}$ will depend both on $\epsilon$ and on $\cos \left(d, d^{1}\right)$. One may for instance find that $m^{1} \cdot d \simeq 1 / 2\left(m^{1} \cdot d^{1}\right)$ for $\cos \left(d, d^{1}\right)=$ 0.5 . The selectivity of the neuron in state $m^{1}$ with respect to a circular environment $\left(d^{1}, \ldots, d^{K}\right)$, such that $\min \cos \left(d^{1}, d^{i}\right)=0.5$, is then lower than 0.5. This should be contrasted with the high selectivity reached by a neuron exposed to all inputs, $d^{1} \ldots d^{K}$.

The one-stimulus environment may be regarded as a case corresponding to rearing the animal in a visual world where only one orientation is present. No controversy remains at present that rearing in such a visual environment results in a cortex in which all visually responsive cells are tuned to the experienced (or nearby) orientations (Blakemore and Cooper, 1970; Hirsch and Spinelli, 1970, 1971; see also Stryker et al., 1978). We see that our theory is in agreement with these findings; moreover, we 
predict that, in such a cortex, the average selectivity of these cells should be lower than normal. Although there is so far no detailed quantitative study on this point, in a recent study, there is some indication that this may indeed be true: "more neurons with normal orientation tuning were found in the kittens that could see all orientations, or at least horizontal and vertical, than in the kittens that had experienced only one orientation" (Rauschecker and Singer, 1981).

\section{IVc. Binocular Input}

We now consider a binocularly driven cell. The firing rate of the neuron at time $t$ becomes

$$
c(t)=m_{r}(t) \cdot d_{r}(t)+m_{l}(t) \cdot d_{l}(t)
$$

with evolution schemes for "right" and "left" states $\boldsymbol{m}_{r}$ and $\boldsymbol{m}_{l}$ straightforward generalizations of equation 4 . Various possibilities now exist for the input $\left(\boldsymbol{d}_{r}, \boldsymbol{d}_{l}\right)$ : one may wish to simulate normal rearing (both $\boldsymbol{d}_{r}$ and $\boldsymbol{d}_{l}$ circular and presumably highly correlated), monocular deprivation, binocular deprivation, etc.

Detailed discussion of the results of simulations under various conditions is given in the next section. The main results are summarized here:

1. In an environment simulating normal binocular rearing, the cell becomes orientation selective and binocular, preferring the same orientation through both eyes.

2. In an environment simulating monocular deprivation, the cell becomes monocular and orientation selective, whatever its initial state.

3. In an environment simulating binocular deprivation, the cell loses whatever orientation selectivity it had but does not lose its responsiveness and, in general, remains driven by both eyes.

\section{Development under Different Rearing Conditions: Comparison of Theory with Classical Experimental Data}

\section{Related Experimental Data}

This brief summary is restricted to area 17 of kitten's cortex. Most kittens first open their eyes at the end of the 1st week after birth. It is not easy to assess whether orientation-selective cells exist at that time in the striate cortex: few cells are visually responsive, and the response's main characteristics are generally "sluggishness" and fatigability. However, it is agreed quite generally that, as soon as cortical cells are reliably visually stimulated (e.g., at 2 weeks), some are orientation selective, whatever the previous visual experience of the animal (cf., Hubel and Wiesel, 1963; Blakemore and Van Sluyters, 1975; Buisseret and Imbert, 1976; Frégnac and Imbert, 1978).

Orientation selectivity develops and extends to all visual cells in area 17 if the animal is reared, and behaves freely, in a normal visual environment (NR): complete "specification" and normal binocularity (about $80 \%$ of responsive cells) are reached at about 6 weeks of age (Frégnac and Imbert, 1978). However, if the animal is reared in total darkness from birth to the age of 6 weeks (DR), then none or few orientation-selective cells are recorded (from 0 to $15 \%$, depending on the authors and the classification criteria); however, the distribution of ocular dominance seems unaffected (Blakemore and Mitchell, 1973; Imbert and Buisseret, 1975; Blakemore and Van Sluyters, 1975; Buisseret and Imbert, 1976; Leventhal and Hirsch, 1980; Frégnac and Imbert, 1978). In animals whose eyelids have been sutured at birth and which are thus binocularly deprived of pattern vision (BD), a somewhat higher proportion (from 12 to $50 \%$ ) of the visually excitable cells are still orientation selective at 6 weeks (and even beyond 24 months of age) and the proportion of binocular cells is less than normal (Wiesel and Hubel, 1965; Blakemore and Van Sluyters, 1975; Kratz and Spear, 1976; Leventhal and Hirsch, 1977; Watkins et al., 1978).

Of all visual deprivation paradigms, putting one eye in a competitive advantage over the other has probably the most striking consequences: monocular lid suture (MD), if it is performed during a "critical" period (ranging from about 3 to about 12 weeks), results in a rapid loss of binocularity, to the profit of the open eye (Wiesel and Hubel, 1963, 1965); then, opening the closed eye and closing the experienced one may result in a complete reversal of ocular dominance (Blakemore and Van Sluyters, 1974). A disruption of binocularity that does not favor one of the eyes may be obtained, for example, by provoking an artificial strabismus (Hubcl and Wiesel, 1965) or by an alternating monocular occlusion, which gives both eyes an equal amount of visual stimulation (Blakemore, 1976). In what follows, we call this uncorrelated rearing (UR).

\section{Theoretical Results}

The aim of this section is to show that the experimental results briefly reviewed above follow from our assumptions if one chooses the appropriate distribution for $\boldsymbol{d}$. The model system now consists of a single binocular neuron. The firing rate of the neuron at time $t$ is given by

$$
\boldsymbol{c}(t)=\boldsymbol{m}_{r}(t) \cdot \boldsymbol{d}_{r}(t)+\boldsymbol{m}_{l}(t) \cdot \boldsymbol{d}_{l}(t)
$$

where the indices $r$ and $l$ refer to right and left eyes, respectively. $\boldsymbol{m}_{r}$ (or $\boldsymbol{m}_{l}$ ) obeys the evolution scheme described by equations 4 to 6 , where $d_{r}$ (or $d_{l}$ ) is substituted for $d$. The two equations are, of course, coupled, since $c(t)$ depends at each $t$ on both $\boldsymbol{m}_{r}(t)$ and $\boldsymbol{m}_{l}(t)$.

The vector $\left(\boldsymbol{d}_{r}, \boldsymbol{d}_{l}\right)$ is a stationary stochastic process, whose distribution is one of the following, depending on the experimental situation one wishes to simulate.

\section{Normal rearing $(N R)$}

$\boldsymbol{d}_{r}(t)=\boldsymbol{d}_{l}(t)$ for all $t$, and $\boldsymbol{d}_{r}$ is circular. (Noise terms that may be added to the inputs may or may not be stochastically independent.)

\section{Uncorrelated rearing (UR)}

$\boldsymbol{d}_{r}$ and $\boldsymbol{d}_{l}$ are i.i.d. (independent identically distributed): they have the same circular distribution, but no statistical relationship exists between them.

\section{Binocular deprivation}

Total light deprivation $(D R)$. The $2 N$ components of $\left(d_{r}, d_{l}\right)$ are i.i.d.: $\boldsymbol{d}_{r}$ and $\boldsymbol{d}_{l}$ are uncorrelated noise terms, $\left(\boldsymbol{d}_{r}, \boldsymbol{d}_{l}\right)=\left(\boldsymbol{n}_{r}, \boldsymbol{n}_{l}\right)$. 
Binocular pattern deprivation $(B D) . d_{r}(t)=\lambda_{r}(t) e$, $\boldsymbol{d}_{l}(t)=\lambda_{l}(t) e$, where $e$ is an arbitrary normalized fixed vector with positive components, and $\lambda_{r}$ and $\lambda_{l}$ are scalar positive valued and i.i.d.

\section{Monocular deprivation (MD)}

$\boldsymbol{d}_{r}$ is circular; $\boldsymbol{d}_{l}$ is a noise term: $\boldsymbol{d}_{l}=\boldsymbol{n}$.

In the NR case, the inputs from the two eyes to a binocular cell are probably well correlated. We therefore assume that they are equal, which is mathematically equivalent. The DR distribution represents dark discharge. The BD distribution deserves a more detailed explanation. In this distribution, it is only the length $\lambda_{r}$ and $\lambda_{l}$ of the vectors $d_{r}$ and $d_{l}$ that varies in time. This length is thought to correspond to the intensity of light coming through each closed eyelid, whereas the direction of the vector in the input space is determined by the constant "unpatterned" vector $e$ (e.g., $e=(1 / \sqrt{N)} \times$ $(1,1, \ldots, 1))$. One may indeed assume that, when light falls on the retina through the closed lids, there is, at any instant of time, high correlation between the firing rates of all retinal ganglion cells on a relatively large region of the retina. Inputs from the two eyes, however, are probably to some extent asynchronous (cf., Kratz and Spear, 1976); hence the $\mathrm{BD}$ distribution.

Simulations of the behavior of the system in these different environments give the following.

$N R$ (Fig. 5a). All asymptotic states are selective and binocular, with matching preferred orientations for stimulation through each eye.

$D R$ (Fig. $5 b$ ). The motion of the state $\left(\boldsymbol{m}_{r}, \boldsymbol{m}_{l}\right)$ resembles a random walk. (The small exponential decay term is necessary here, too, in order to prevent large fluctuations.) The two tuning curves ${ }^{9}$ therefore undergo random fluctuations that are essentially determined by the second order statistics of the input $d$. As can be seen from the figure, these fluctuations may result sometimes in a weak orientation preference or unbalanced ocular dominance. However, the system never stays in such states very long; its average state on the long run is perfectly binocular and non-oriented. Moreover, whatever the second order statistics of $\boldsymbol{d}$ and the circular environment in which tuning curves are assessed, a regular unimodal orientation tuning curve is rarely observed, and selectivity has never exceeded 0.6 . Thus, we may conclude that orientation selectivity as observed in the NR case (both experimental and theoretical) cannot be obtained from purely random synaptic weights. It is worth mentioning here that prolonged dark rearing has been reported to increase response variability (Leventhal and Hirsch, 1980); a similar observation was made by Frégnac and Bienenstock (1981). ${ }^{10}$

$B D$ (Fig. 5c). Unlike the DR case, the state converges

\footnotetext{
${ }^{9}$ The circular environment which serves to assess the orientation tuning curves is now, in a sense, arbitrary, since it is not at all used in the development period. The same remark applies, of course, to the BD case.

${ }^{10}$ In Figure $1 B$ of Frégnac and Bienenstock (1981), which shows averaged orientation tuning curves of a cell recorded in an 86-day-old DR cat, the selectivity is 0.58 at the beginning of the recording session and 0.28 at the end.
}

(as may easily be proved mathematically). Although there exist both monocular and binocular stable equilibrium points, the asymptotic state is generally monocular if the initial state is taken as 0 . The orientation tuning curve then is determined essentially by the relative geometry of the fixed arbitrary vector $e$ and the arbitrary circular environment which serves to assess the tuning curve. Fine unimodal tuning, therefore, is not to be expected.

$M D$ (Fig. $5 d$ ). The only stable equilibrium points are monocular and selective. The system converges to such states whatever the initial conditions. In particular, this accounts for reverse suture experiments (Blakemore and Van Sluyters, 1974; Movshon, 1976).

$U R$ (Fig. 5e). This situation is, in a sense, similar to the BD one: the state converges, but monocular as well as binocular equilibria exist. As in the $\mathrm{BD}$ case, the asymptotic state generally observed with $m_{r}(0)=m_{l}(0)$ $=0$ is monocular. (This should be attributed to the mismatched inputs from the two eyes, as is done by most authors.) In this case, however, asymptotic states are selective, and when they are binocular, preferred orientations through each eye do not necessarily coincide. It should be mentioned here that Blakemore and Van Sluyters (1974) report that, after a period of alternating monocular occlusion, the remaining binocular cells may differ in their preferred orientations for stimulation through each eye.

These results are in agreement with the classical experimental data in the domain of visual cortex development. Most of them can be obtained fairly easily, with no need of further simulations, as a consequence of the convergence to selective states in the case of a monocularly driven neuron in a circular environment (section IVa).

Some intriguing properties of our theory are more subtle, however, and, in addition to contributing to the results above, provide the opportunity for applications to more complicated experimental paradigms and for new tests. As an example, it is shown in "Appendix C" that, in the MD case, the degree of monocularity of the cortical cell is correlated with its orientation selectivity as well as the diversity of inputs to the open eye. These unexpected predictions agree well with the observation by Cynader and Mitchell (1980) and Trotter et al. (1981) that, after a brief period of monocular exposure, oriented cells are more monocular than non-oriented ones as well as the observation of Rauschecker and Singer (1981) that an open eye with restricted inputs leads to cells oriented to the restricted input that are driven less monocularly than usual. A summary of theoretical results is given below.

\section{Discussion}

We have proposed a new mathematical form for synaptic modification and have investigated its consequences on the development of selectivity in cortical neurons. In addition, we have provided a definition of the notion of selectivity with respect to a random variable that might be applied in many different situations (in the domain of development of sensory systems, for example, selectivity of binocular neurons to retinal disparity, etc.). In its application to visual cortex, our theory is in agree- 

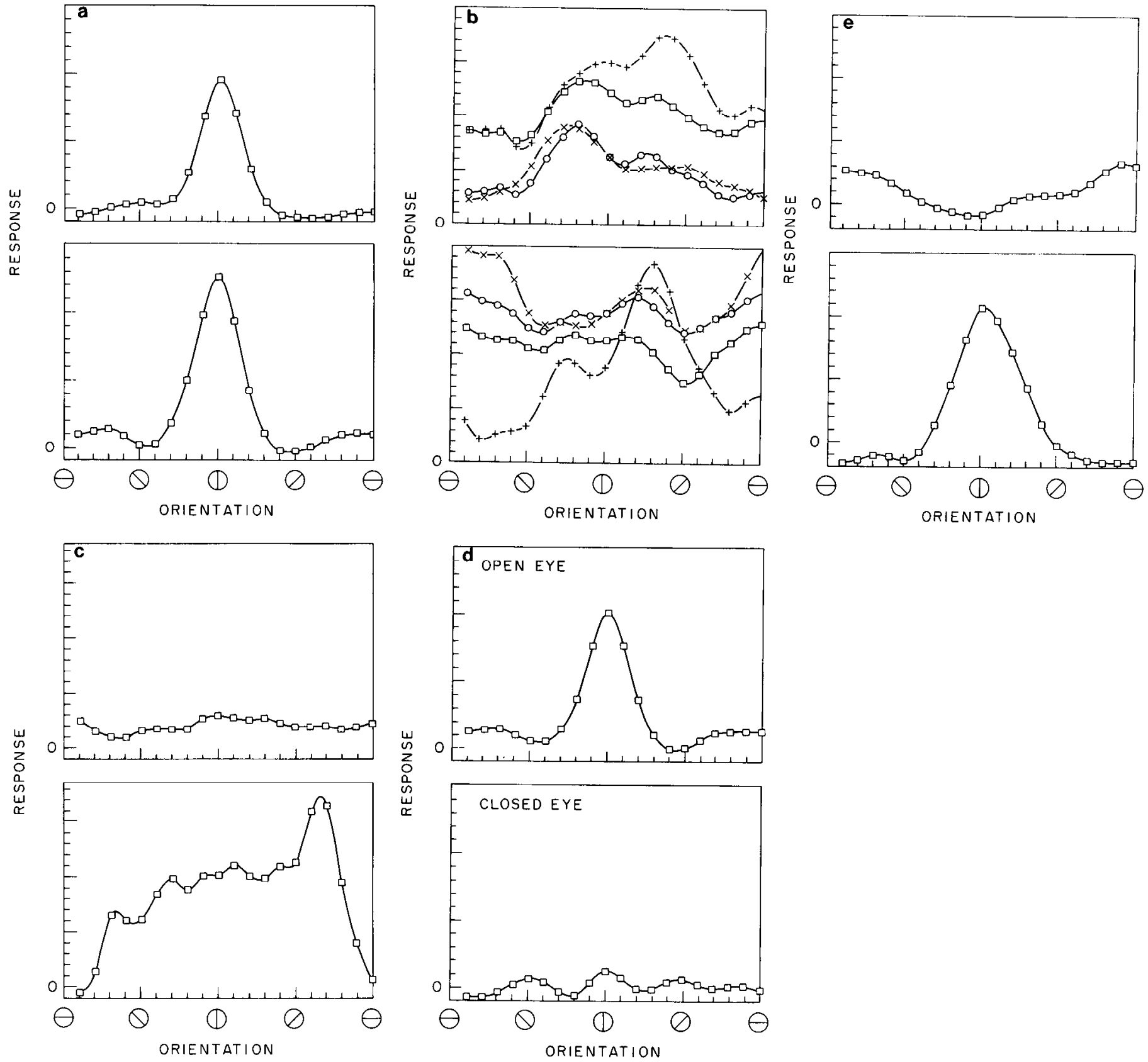

Figure 5. Results of computer simulations corresponding to various rearing conditions. In these simulations, the upper and lower panels show cell responses to stimuli from the two eyes. $a$, Normal (NR). The cell's response is binocular and selective. $b$, Dark rearing (DR). There is no stable selectivity in the cell's response. The response curve fluctuates randomly. The cell is, on the average, driven binocularly. $c$, Binocular deprivation (BD). The cell reaches a final state corresponding to the arbitrary vector which corresponds to a diffuse input to the retina. The cells sometimes are driven monocularly. This is somewhat analogous to $e$ below. $d$, Monocular deprivation (MD). The cell's response is monocular and selective. $e$, Uncorrelated rearing (UR). Both binocular and monocular selective final states are observed.

ment with the classical experimental results obtained over the last generation and offers a number of new predictions, some of which can be tested experimentally. This may lead to the identification of the parameters of the theory and provide indications as to the biochemical mechanisms underlying cortical plasticity.

In a broader context, we may regard our form of synaptic modification as a specific correlation modifica- tion of a hebbian type. The great majority of models on a synaptic level in domains such as pattern recognition, task learning, or associative memory ${ }^{11}$ (which are less accessible to direct neurophysiological experimentation)

${ }^{11}$ Notice, for instance, the analogy between states of maximum selectivity as defined here and the optimal associative mappings of Kohonen (1977). 
use schemes of a hebbian type with some success. Thus, we are led to conjecture that some form of correlation modification is a very general organizational principle that manifests itself in visual cortex in a manner that is accessible to experiment.

Although synaptic competition is a natural consequence of Hebb's principle, it may be given various mathematical forms. A distinction was made in section II between spatial competition-the form commonly accepted by theoreticians as well as experimentalists-and temporal competition-a new form proposed in this work. Competition is said to be purely spatial, or to take place between converging afferents, if the sign of $\dot{m}_{j}(t)$ is determined by a comparison of the firing rate $d_{j}(t)$ with firing rates $d_{k}(t)$ in the other afferents to the neuron at the same time, $t$. In some schemes (e.g., von der Malsburg, 1973), the sign of $\dot{m}_{j}(t)$ also depends on the value of the synaptic efficacy $m_{j}(t)$ relative to $m_{k}(t), 1 \leq k \leq$ $N$. Formally then, we might characterize competition as purely spatial if

$$
\operatorname{sign}\left(\dot{m}_{j}(t)\right)=F\left(\frac{d_{j}(t)}{d_{1}(t)}, \ldots, \frac{d_{j}(t)}{d_{N}(t)}, \frac{m_{j}(t)}{m_{1}(t)}, \ldots, \frac{m_{j}(t)}{m_{N}(t)}\right)
$$

On the other hand, we say that competition is purely temporal, or takes place between incoming patterns, if the sign of $\dot{m}_{j}(t) / d_{j}(t)$ is independent of $j$ and is determined by a relationship between the postsynaptic neuron's firing rates, $c(t)$ and $c\left(t^{\prime}\right), t^{\prime}<t$ :

$$
\operatorname{sign}\left(\frac{\dot{m}_{j}(t)}{d_{j}(t)}\right)=F\left(c(t) ; c\left(t^{\prime}\right)\right), \quad t^{\prime}<t, \quad j=1 \ldots N
$$

In this work, the modification threshold, $\theta_{M}$, is given as a function of $\bar{c}$, an average of $c\left(t^{\prime}\right)$ over a relatively long time period preceding $t$, thus satisfying equation 10 .

One may, of course, imagine hebbian schemes of a mixed type, involving both spatial and temporal competition. ${ }^{12}$ However, the distinction is useful since the performance of the scheme seems to be highly dependent on which of the two classes it is in. This is most clearly seen in the development of selectivity.

In the temporal version used here, asymptotic states are of maximum selectivity with respect to the experienced environment $\boldsymbol{d}$, independent of the geometry of $\boldsymbol{d}$. This was rigorously proven analytically in some cases (theorem 1; section III) and conjectured on the basis of numerical results in other cases (circular environments; section IV). In contrast with this, we conjecture that, in any model using pure spatial competition, behavior will usually depend on the geometry of the environment. In any case, maximum selectivity is not reached if the patterns in the environment are not sufficiently separated from one another. This is illustrated in "Appendix B" for one particular model using spatial competition between converging afferents.

\footnotetext{
${ }^{12}$ More complicated temporal or mixed spatiotemporal schemes are possible and some such have been proposed. For example, Sejnowski (1977a) has suggested a form of modification in which the change of the $j$ th synaptic strength involves the co-variance between the $j$ th fiber and postsynaptic activities. In addition, interaction between neurons (such as lateral inhibition) can increase selectivity (see, for example, Nass and Cooper, 1975 and "Appendix D").
}

We further note that selectivity, as was shown in section V, does not develop in a "pure noise" environment (the distribution termed DR). Some kind of patterned input is required.$^{13}$ It follows that, at this level of organization of connectivity, information is being transferred from the environment to the system. This may shed some light on what has been known for a long time as the innate/learned controversy in visual cortex. Our results suggest that this dichotomy is, at best, misleading. The system's potential developmental ability-its evolutive power-may indeed be determined genetically; yet selectivity has no meaning if it does not refer to a given structured environment that determines the final organization of the system. ${ }^{14}$

The present work, however, makes no assumption concerning the initial state of cortex (e.g., the presence or absence of selectivity at eye opening). This question, still a subject of controversy (see Pettigrew, 1978), must be settled experimentally. Further, although we here assume that all synapses are equally modifiable, it could easily be the case that there is variation in modifiabilityeven one that is time dependent-and that, for example, some of the initial state information including some orientation selectivity is contained in a skeleton of synapses that is less modifiable. ${ }^{15}$ Such assumptions can easily be incorporated in fairly obvious extensions of our theory and would, of course, result in the modification of some details of our results. The principal results of our theory, applied to visual cortical neurons and assuming that they are all equally modifiable according to equations 4 to 7 , are summarized now. These are either in agreement with existing experimental data or are new and somewhat unexpected consequences of our theory.

\section{Summary of Theoretical Results-New Predictions}

\section{Monocularly driven neurons}

(1) A monocularly driven neuron in a "normal" (patterned) environment becomes selective. The precise pattern to which it becomes selective is determined at random if the initial selectivity is 0 or may be biased toward a particular pattern if there is a built-in preference for this pattern.

(2) This same neuron in various deprived environments evolves as follows.

Pure noise. The neuron becomes less selective but

${ }^{13}$ Pure noise and circular environments may be regarded as two extreme cases: the first totally lacks structure, whereas the second is highly organized. Intermediate cases (i.e., environments consisting of the sum of a noise process and of a circular process) also have been investigated (see, for example, Bienenstock, 1980). There it is shown that the asymptotic selectivity directly depends on a parameter that measures the degree of structure of the environment.

${ }^{14}$ We note, further, that the mechanism of synaptic modification that we have proposed leads both to what are sometimes called "selective" and "instructive" effects (depending on the structure of the environment and the genetic initial state). Thus, as is already suggested by Rauschecker and Singer (1981), this dichotomy is obscured, or does not appear at all, at the synaptic level.

${ }^{15}$ This skeleton might consist primarily of the contralateral pathway and favor the development of the orientation preference for horizontally and vertically oriented stimuli (see, for example, Frégnac, 1979). 
continues to be (somewhat) responsive. It may show an orientation preference, but this is relatively unstable.

Exposure to a single pattern (such as vertical lines). The neuron comes to respond preferentially to the single pattern but with less selectivity (less sharply tuned) than if all orientations were present in the environment. This last is a natural consequence of temporal competition between incoming patterns and can provide a good test of our theory. ${ }^{16}$

(3) Inhibitory synapses are required to produce maximum selectivity. If such inhibitory connections are arbitrarily set equal to 0 , selectivity diminishes.

\section{Binocularly driven neurons}

(1) A binocularly driven neuron in a "normal" (patterned) environment becomes selective and binocular. It is driven selectively by the same pattern from both eyes.

(2) This same binocularly driven neuron in various deprived environments evolves as follows.

Uncorrelated patterned inputs to both eyes. The neuron becomes selective, often monocularly driven; if the neuron is binocular, sometimes it is driven by different patterns from the two eyes.

Patterned input to one eye, noise to the other (monocular deprivation). The neuron becomes selective and generally driven only by the open eye. There is a correlation between selectivity and binocularity. The more selective the neuron becomes, the more it is driven only by the open eye. A non-selective neuron tends to remain binocularly driven. This correlation is due, in part, to the fact that it is the same mechanism of synaptic change that serves to increase both the selectivity and ocular dominance of the open eye. However (as shown in "Appendix C"), there is also a subtler connection: it is the non-preferred inputs from the open eye accompanied by noise from the closed eye that drive the neuron's response to the closed eye to 0 . Thus, for example, if the visual cnvironment were such that there were mostly preferred inputs to the open eye, even a selective cell would remain less monocular. (It should prefer the open eye but remain somewhat driven by the closed eye.) As another example, a kitten dark-reared to the age of about 42 days (when there remain few or no specific cells) and then given monocular exposure to nonpatterned input would retain more binocularly driven cells than a similar animal given patterned input. ${ }^{17}$

\footnotetext{
${ }^{16}$ In addition, the principle of temporal competition suggests an experimental paradigm that could be used to increase the selectivity of a cortical neuron while recording from the same neuron. The paradigm consists of controlling the postsynaptic activity of the neuron while presenting sequentially in its receptive field two stimuli, $A$ and $B$. Stimulus A (or B) should be associated with a high (or low) instantaneous firing rate in such a way as to keep the cell's mean firing rate at its original value. We predict that the cell will prefer stimulus A eventually (i.e., exhibit selectivity with respect to the discrete environment consisting of $A$ and $B$ ). Moreover, we predict that presentation of stimulus A alone will lead to less selectivity. An experiment based on this paradigm is currently being undertaken by one of us (E. L. B.) in collaboration with Yves Frégnac.

${ }^{17}$ In this situation, one might have to distinguish between short and long monocular exposures. In very long monocular exposures, the decay term of equation $4(-\epsilon m(t))$ eventually could produce decay of junctions from the closed eye independent of the effect discussed above.
}

Noise input to both eyes (dark rearing or binocular deprivation). The neuron remains non-selective (or loses its selectivity) and diminishes its responsiveness but remains binocularly driven (in contrast to the situation in monocular deprivation).

These theoretical conclusions are consistent with experimental data on increases and decreases in selectivity, data concerning changes in ocular dominance in various rearing conditions, as well as data from more complicated paradigms. Although there are indications in recent work that some of the new predictions are in agreement with experimental results, they provide the opportunity for tests of subtler aspects of the theory.

In conclusion, we note that a precise application of our theory to certain complicated experimental situations would probably require inclusion of some anatomical details, interneuronal interactions, as well as a statement of what information is innate and which synapses are modifiable. $^{18}$

\section{Appendix A: Biochemical Mechanism for Temporal Competition}

It is probably premature to propose a detailed physiological mechanism for a mathematical synaptic modification algorithm: too many possibilities exist with no present experimental test to decide among them. However, we propose the following as a possible example.

The dependence of our modification threshold upon the mean postsynaptic activity, which regulates the individual neuron modification in an overall manner, might be the result of a physiological mechanism within the framework proposed by Changeux et al. (1973). Their basic hypothesis is that receptor protein on the postsynaptic membrane exists in two states, one labile and the other stable; selective stabilization of the receptor takes place during development in an activity-dependent fashion. The quantity of labile receptor available for stabilization is determined by the neuron's average activity; that is, labile receptor is not synthesized anymore when the neuron's activity is high for a relatively long period of time $\left(\bar{c} \gg c_{0}\right)$ (cf., Changeux and Danchin (1976): "The activity of the postsynaptic cell is expected to regulate the synthesis of receptor.").

Our hypothesis that, during the period when competition really takes place (i.e., when $\bar{c}$ is of the order of $c_{0}$ in equation 7), the sign of the modification is determined by the instantaneous activity, $c$, relative to its mean, $\bar{c}$, requires that a single message, the instantaneous activity, be fed back from the site of integration of the incoming message to the individual synaptic sites, on a rapid time scale (i.e., much faster than the one involved in the overall regulation mechanism). This might be contrasted with the assumption implicit in most spatial competition models, namely, that a chemical substance is redistributed between all subsynaptic sites (cf., the principle of conservation of total synaptic strength (von der Malsburg, 1973)).

${ }^{18}$ This last might be treated as, for example, in the work of Cooper et al. (1979). 


\section{Appendix B: von der Malsburg's Model of} Development of Orientation Selectivity

A model of development of orientation selectivity using an evolution scheme of the spatial type may be found in the work of von der Malsburg (1973). We present here a brief analysis of this model in view of the definition given in section II. We first show that the type of competition implied by this model is indeed, formally, the spatial one. Next, we investigate the behavior of the system in the simple situation of theorem 1 in section III (i.e., for a two-pattern environment, with the dimension of the system being $N=2$ ). We will show why the assumption that is made of nonlinearity of the integrative power is a necessary one. Finally, we prove that the class of twopattern environments $\boldsymbol{d}$ in which the system behaves nicely (i.e., the state is asymptotically selective with respect to $d$ ) is defined by a condition of the type $0<$ $\cos \left(d^{1}, d^{2}\right)<a$, where $d^{1}$ and $d^{2}$ are the two patterns in $d$, and $\boldsymbol{a}$ is a constant strictly less than 1, which actually depends on the nonlinearity of the integrative power (i.e., on its threshold $\Theta$ ).

For the purpose of our analysis, we consider a single "cortical" neuron whose integrative and evolutive power are, in our notation, the following:

$$
c(t)=(\boldsymbol{m}(t) \cdot d(t))^{*}
$$

with

$$
\begin{gathered}
u^{*}=\left\{\begin{array}{lll}
u-\Theta & \text { if } & u>\Theta \\
0 & \text { if } & u<\Theta
\end{array}\right. \\
\boldsymbol{m}_{j}(t+1)=\gamma(t+1)\left(\mathbf{m}_{j}(t)+h \boldsymbol{c}(t) \boldsymbol{d}_{j}(t)\right) \quad j=1, \ldots, N
\end{gathered}
$$

with $h$ a small positive constant and $\gamma(t+1)$ such that:

$$
\sum_{j=1}^{N} \boldsymbol{m}_{j}(t+1)=\sum_{j=1}^{N} \boldsymbol{m}_{j}(t)=s
$$

The integrative power is thus nonlinear with threshold $\Theta$. The normalizing factor $\gamma(t+1)$ in the evolution equation B3 keeps the sum of synaptic weights constant and equal to $s$. All variables are positive.

Our analysis will be carried out on this reduced version of von der Malsburg's model: we simply ignore the fixed intracortical connections assumed there, for these are clearly not sufficient to tune the system to a selective state if individual neurons do not display this property already. As is clearly stated by the author himself, the ability to develop selectivity is an intrinsic property of individual neurons, the intracortical connections being there to organize orientation preference in a coherent way in cortex. (This is also the viewpoint in the present work: see "Appendix D.") Notice that this is by no means a contradiction to the fact that, in the final state, intracortical connections, particularly the inhibitory ones, significantly contribute to the selectivity of each neuron.

A straightforward calculation shows that equations B3 and $\mathrm{B} 4$ are equivalent to the following.

$$
\begin{cases}m_{j}(t+1)-m_{j}(t)=K(t)\left(d_{j}(t) / d(t)-m_{j}(t) / s\right) & j=1, \ldots, N \\ K(t)=\operatorname{shc}(t) d(t) /(s+h c(t) d(t)) & \end{cases}
$$

where

$$
\boldsymbol{d}(t)=\sum_{j=1}^{N} \boldsymbol{d}_{j}(t)
$$

(In the simulations, $\boldsymbol{d}(t)$ is actually a constant.)

Thus, according to equation B5, the sign of the change of $\boldsymbol{m}_{j}$ at time $t$ does not depend on the postsynaptic activity $c(t)$ but on the $j$ th fiber activity $\boldsymbol{d}_{j}(t)$. This is clearly spatial competition as is suggested by the conservation law (equation $B 4$ ).

We now investigate the behavior of system B5 in a two-pattern environment: $P\left[\boldsymbol{d}=d^{1}\right]=P\left[\boldsymbol{d}=d^{2}\right]=0.5$. For this purpose, we slightly modify the original setup: there, the dimension is relatively high $(N=19)$, but the firing frequencies in the afferent fibers are discretely valued (i.e., $d_{j}=0$ or $1, j=1, \ldots, N$ ). Here, we take $N$ $=2$, with $d_{1,2}$ allowed to take any value between 0 and 1 . By doing so, we still get a broad range of environments $\left(\cos \left(d^{1}, d^{2}\right)\right.$ may assume any value between 0 and 1$)$, but the analysis is made considerably easier. To further simplify, we characterize $\boldsymbol{d}$ by a single parameter $0<\delta$ $<1$ by writing $d^{1}=(1, \delta), d^{2}=(\delta, 1)$. Thus, $\cos \left(d^{1}, d^{2}\right)$ $=2 \delta /\left(1+\delta^{2}\right)$. We also set $s=1$.

Under these circumstances, averaging the evolution equation B5 with respect to $d$ leads to the following:

$$
\begin{gathered}
E\left[m_{j}(t+1)-m_{j}(\mathrm{t})\right]= \\
1 / 2 h\left(2 m_{j}(\mathrm{t})-1\right)(\Theta(1+\delta)-2 \delta), \quad \mathrm{j}=1,2
\end{gathered}
$$

To obtain equation $\mathrm{B} 6$, it has been assumed that both inputs yield above threshold responses (i.e., $m \cdot d^{1}$ and $m \cdot d^{2}>\Theta$ ). Higher order terms in $h$ have been ignored.

We see that the behavior of the system is determined by the sign of the quantity $\Theta(1+\delta)-2 \delta$. Notice that, since $s=1, \Theta$ cannot be arbitrarily high: in order that states $m$ exist such that $m \cdot d^{1}$ and $m \cdot d^{2}>\Theta$, one has to assume that $\Theta<(1+\delta) / 2$.

It follows from equation B 6 that, for $\delta$ such that $\Theta(1+\delta)-2 \delta<0$, there is one attractor of selectivity 0 , namely $(0.5,0.5)$. When $\delta$ gets smaller and $\Theta(1+\delta)-2 \delta$ becomes positive, the solution bifurcates into two attractors of maximum selectivity. We thus conclude that:

1. If the neuron's integrative power is linear (i.e., $\Theta=0$ ), the asymptotic state is non-selective. (When $\Theta=0$ and $d^{1}$ and $d^{2}$ are orthogonal (i.e., $\delta=0$ ), the first order term in $h$ vanishes, yet the second order term also leads to the non-selective fixed point.)

2. Given a fixed $0<\Theta<1$, the environments $d$ that are acceptable to the system are those which satisfy $\delta<\Theta /(2-\Theta)$, which is equivalent to a condition of the type $\cos \left(d^{1}, d^{2}\right)<a$ with $a$ strictly less than 1 . (Notice that, in the actual simulations, $d$ consists of nine stimuli that are indeed well separated from one another, since $\min _{i} \cos \left(d^{1}, d^{i}\right)=1 / 7$.)

\section{Appendix C: Correlation between Ocular Dominance and Selectivity in the Monocular Deprived Environment}

Consider the MD environment in section V: it is defined by $\left(d_{r}, n\right)$, where $d_{r}$ is circular and $n$ is a "pure noise" vector. We will prove that the state $\left(m_{r}^{*}, 0\right)$ is stable in this environment provided that $m_{r}^{*}$ is a stable selective state in the environment $\boldsymbol{d}_{r}$. 
Let $\left(x_{r}, x_{l}\right)$ be a small perturbation from equilibrium. The motion at point $\left(m_{r}^{*}+\boldsymbol{x}_{r}, \boldsymbol{x}_{l}\right)$ is given by:

$$
\begin{gathered}
\dot{\boldsymbol{x}}_{r}=\phi\left(m_{r}^{*} \cdot \boldsymbol{d}_{r}+\boldsymbol{x}_{r} \cdot \boldsymbol{d}_{r}+\boldsymbol{x}_{i} \cdot \boldsymbol{n}, m_{r}^{*} \cdot \bar{d}_{r}+\boldsymbol{x}_{r} \cdot \bar{d}_{r}\right) \boldsymbol{d}_{r} \\
\dot{\boldsymbol{x}}_{l}=\phi\left(m_{r}^{*} \cdot \boldsymbol{d}_{r}+\boldsymbol{x}_{r} \cdot \boldsymbol{d}_{r}+\boldsymbol{x}_{i} \cdot \boldsymbol{n}, m_{r}^{*} \cdot \bar{d}_{r}+\boldsymbol{x}_{r} \cdot \bar{d}_{r}\right) \boldsymbol{n}
\end{gathered}
$$

where we assume that the noise has 0 mean.

We analyze separately, somewhat informally, the behavior of the two equations. The stability of equation C1r is immediate from the stability of the selective state $m_{r}^{*}$ in the circular environment $\boldsymbol{d}_{r}$. To analyze equation $\mathrm{C} 1 \mathrm{l}$, we divide the range of the right eye input $\boldsymbol{d}_{r}$ into three classes:

1. $\boldsymbol{d}_{r}$ is such that $m_{r} \cdot \boldsymbol{d}_{r}$ is either far above threshold, $\theta_{M}$, and therefore $\phi\left(m_{r} \cdot d_{r}, m_{r} \cdot \bar{d}_{r}\right)>0$, or far below threshold, $\theta_{M}$ (but still positive), and therefore $\phi\left(m_{r} \cdot \boldsymbol{d}_{r}, m_{r} \cdot \overline{\boldsymbol{d}}_{r}\right)<0$. This case might occur before $m_{r}$ has reached a stable selective state, $m_{r}^{*}$.

2. $\boldsymbol{d}_{r}$ is such that $m_{r}^{*} \cdot \boldsymbol{d}_{r}$ is near threshold, $\theta_{M}$, and therefore, $\phi\left(m_{r}^{*} \cdot \boldsymbol{d}_{r}, m_{r}^{*} \cdot \bar{d}_{r}\right) \simeq 0$.

3. $\boldsymbol{d}_{r}$ is such that $m_{r}^{*} \cdot d_{r} \simeq 0$, again resulting in $\phi\left(m_{r}^{*} \cdot \boldsymbol{d}_{r}, m_{r}^{*} \cdot \bar{d}_{r}\right) \simeq 0$.

For the first class of inputs, the sign of $\phi$ is determined by $\boldsymbol{d}_{r}$ alone, hence equation $\mathrm{Cll}$ is the equation of a random walk. To investigate the behavior of equation C1l in the two other cases, we neglect the term $\boldsymbol{x}_{r}$ and linearize $\phi$ around the relevant one of its two zeros. It is easy to see that case 2 yields

$$
\dot{x}_{l} \simeq \epsilon_{1}\left(x_{l} \cdot n\right) n
$$

whereas, in case 3 , one obtains

$$
\dot{\boldsymbol{x}_{l}} \simeq-\epsilon_{2}\left(\boldsymbol{x}_{i} \cdot \boldsymbol{n}\right) \boldsymbol{n}
$$

where $\epsilon_{1}$ and $\epsilon_{2}$ are positive constants, measuring, respectively, the absolute value of the slope of $\phi$ at the modification threshold and at zero. ${ }^{8}$

Since $n$ is a noise-like term, its distribution is presumably symmetric with respect to $\boldsymbol{x}_{l}$ so that averaging equations $\mathrm{C} 2$ and $\mathrm{C} 3$ yields, respectively

$$
\begin{gathered}
\dot{x_{l}} \simeq \epsilon_{1} \overline{n_{0}^{2}} x_{l} \\
\dot{x_{l}} \simeq-\epsilon_{2} \overline{n_{0}^{2}} x_{l}
\end{gathered}
$$

where $\overline{n_{0}^{2}}$ is the average squared magnitude of the noise input to a single synaptic junction from the closed eye.

We thus see that input vectors from the first class move $x_{l}$ randomly, inputs from the second class drive it away from 0 , whereas inputs from the third drive it toward 0. In the case where the range of $d_{r}$ is a set of $K$ linearly independent vectors and $m_{r}^{*}$ is of maximum selectivity, $(K-1) / K$, case 1 does not occur at all. (The random contribution occurs only before the synaptic strengths from the open eye have settled to one of their fixed points). Case 2 occurs only for one input, e.g., $d_{r}^{1}$, with $m_{r}^{*} \cdot \mathrm{d}_{r}^{1}$ exactly equal to threshold, $\dot{\theta}_{M}$, and case 3 occurs for the other $K-1$ vectors which are all orthogonal to $m_{r}^{*}$. In the general case ( $\boldsymbol{d}_{r}$ any circular environment), the more selective $m_{r}^{*}$ with respect to $\boldsymbol{d}_{r}$, the higher the proportion of inputs belonging to class 3 , the class that yields equation $\mathrm{C} 5$ (i.e., that brings $\boldsymbol{x}_{l}$ back to 0 ).

The stability of the global system still depends on the ratio of the quantities $\epsilon_{1}$ and $\epsilon_{2}$ as well as on the statistics of the noise term $n$ (e.g., its mean square norm). We may, however, formulate two general conclusions. First, under reasonable assumptions ( $\epsilon_{1}$ of the order of $\epsilon_{2}$ and the mean square norm of $\boldsymbol{n}$ of the same order as that of $\boldsymbol{d}_{r}$ ), $\boldsymbol{x}_{l}=0$ is stable on the average for a selective $m_{r}^{*}$. Second, the residual fluctuation of $\boldsymbol{x}_{l}$ around zero, essentially due to inputs $\boldsymbol{d}_{r}$ in classes 1 and 2, is smaller for highly selective $m_{r}^{*}$ values than it is for mildly selective ones.

Thus, one should expect that, in a monocularly deprived environment, non-selective neurons tend to remain binocularly driven. In addition, since it is the nonpreferred inputs from the open eye accompanied by noise from the closed eye (case 3 ) that drive the response to the closed eye to 0 , if inputs to the open eye were restricted to preferred inputs (case 2), even a selective cell would remain less monocular.

\section{Appendix D: Many-neuron Systems}

It is very likely that interactions between cortical neurons play an important role in overall cortical function as well, perhaps, as in selectivity of individual cortical cells (Creutzfeldt et al., 1974; Sillito, 1975). The development of selectivity then might be regarded as a many-neuron problem. Since the underlying principles put forward in this work are stated most clearly at the single unit level (where a more complete analysis is also possible), we have chosen this description. However, the methods employed are also applicable to a system of many cortical neurons interacting with one another. Most important, the result that stable equilibria in a stationary environment are selective with respect to their environment can be taken over to the many-neuron system.

Consider such a system in a stationary external environment. The state of each cortical neuron now has two parts: one relative to the geniculocortical synapses, the other to the cortico-cortical ones. The environment of the neuron is no longer stationary, for the states of all other cortical neurons in the system evolve. Yet, when the system reaches global equilibrium, which will occur under reasonable assumptions, each individual environment becomes stationary. The single unit study then allows us to state that, at least in principle (we do not know a priori that each environment is circular), the state of each neuron is selective with respect to its own individual environment.

In practice, formulation of the many-neuron problem poses two questions. First, the integrative power of the system should be specified. Since the system includes cortico-cortical loops, it is not obvious what the response to a given afferent message should be. The two major alternatives are: $(a)$ stationary cortical activity is reached rapidly (i.e., before the afferent message changes) and (b) relevant cortical activity is transitory. The second question concerns the evolution of cortico-cortical synaptic strengths: should these synapses be regarded as modifiable at all, and if yes, how? von der Malsburg (1973) assumes alternative $a$ above and proposes fixed connectivity patterns, short range excitatory and longer range inhibitory.

We have performed a simulation of a many-neuron system using the much simpler (and probably more nat- 


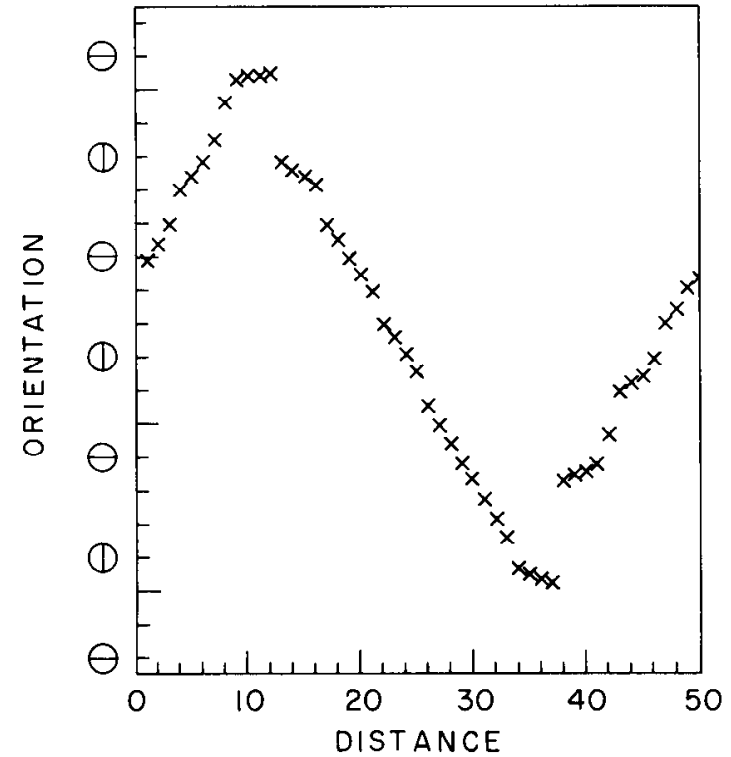

Figure 6. A regular distribution of preferred orientation in a one-dimensional cortex. The system is an array of 50 "cortical" cells arranged in a cyclic way (cell 1 and cell 50 are neighbors) and interconnected according to a fixed short-range-excitationlong-range-inhibition pattern. (Coefficients of interactions are, as a function of increasing intercell distance: $0.4,0.4,-0.2$, $-0.4,0, \ldots)$. The environment $d$ of the system is the usual circular one. Integrative and evolutive powers are described in the text. When the system reaches equilibrium, one has $0.73 \leq \operatorname{Sel}_{d}\left(m_{i}\right) \leq 0.77$ for all $i$ values between 1 and 50 . The diagram shows preferred orientation as a function of cortical coordinate.

ural) assumption $b$ above. (1) Only monosynaptically and disynaptically mediated components of the afferent message are taken into account for the computation of each cortical neuron's activity before modification is performed and a new stimulus is presented. (2) Intracortical connections are fixed and spatially organized as in the work of von der Malsburg (1973). (3) The state of each neuron evolves according to equations 6 and 7 of the present work. The results are the following. (1) The system's state converges. (2) At equilibrium, each neuron stands in a selective state with respect to the environment. (3) Preferred orientation-when the environment is a circular one-is a piecewise continuous function of cortical distance (Fig. 6). (4) In the final equilibrium state, the intracortical synapses contribute along with the geniculocortical ones to produce the selectivity.

\section{References}

Anderson, J. A. (1970) Two models for memory organization using interacting traces. Math. Biosci. 8: 137-160.

Anderson, J. A. (1972) A simple neural network generating an interactive memory. Math. Biosci. 14: 197-200.

Bienenstock, E. (1980) A theory of development of neuronal selectivity. Doctoral thesis, Brown University, Providence, RI.

Blakemore, C. (1976) The conditions required for the maintenance of binocularity in the kitten's visual cortex. J. Physiol. (Lond.) 261: 423-444.

Blakemore, C., and G. F. Cooper (1970) Development of the brain depends on the visual environment. Nature 228: 477478.
Blakemore, C., and D. E. Mitchell (1973) Environmental modification of the visual cortex and the neural basis of learning and memory. Nature 241: 467-468.

Blakemore, C., and R. C. Van Sluyters (1974) Reversal of the physiological effects of monocular deprivation in kittens. Further evidence for a sensitive period. J. Physiol. (Lond.) 237: 195-216.

Blakemore, C., and R. C. Van Sluyters (1975) Innate and environmental factors in the development of the kitten's visual cortex. J. Physiol. (Lond.) 248: 663-716.

Brindley, G. S. (1969) Nerve net models of plausible size that perform many simple learning tasks. Proc. R. Soc. Lond. (Biol.) 174: 173-19i.

Buisseret, P., and M. Imbert (1976) Visual cortical cells. Their developmental properties in normal and dark reared kittens. J. Physiol. (Lond.) 255: 511-525.

Buisseret, P., E. Gary-Bobo, and M. Imbert (1978) Ocular motility and recovery of orientational properties of visual cortical neurones in dark-reared kittens. Nature 272: 816817.

Changeux, J. P., and A. Danchin (1976) Selective stabilization of developing synapses as a mechanism for the specification of neuronal networks. Nature 264: 705-712.

Changeux, J. P., P. Courrège, and A. Danchin (1973) A theory of the epigenesis of neuronal networks by selective stabilization of synapses. Proc. Natl. Acad. Sci. U. S. A. 70: 29742978.

Cooper, L. N. (1973) A possible organization of animal memory and learning. In Proceedings of the Nobel Symposium on Collective Properties of Physical Systems, B. Lindquist and S. Iindquist, eds., Vol., 24, pp. 252-264, Academic Press, New York.

Cooper, L. N., F. Lieberman, and E. Oja (1979) A theory for the acquisition and loss of neuron specificity in visual cortex. Biol. Cybern. 33: 9-28.

Creutzfeldt, O. D., U. Kuhnt, and L. A. Benevento (1974) An intracellular analysis of visual cortical neurones to moving stimuli: Responses in a cooperative neuronal network. Exp. Brain Res. 21: 251-274.

Cynader, M., and D. E. Mitchell (1980) Prolonged sensitivity to monocular deprivation in dark-reared cats. J. Neurophysiol. 43: $1026-1040$.

Frégnac, Y. (1979) Development of orientation selectivity in the primary visual cortex of normally and dark reared kittens. Biol. Cybern. 34: 187-204.

Frégnac, Y., and E. Bienenstock (1981) Specific functional modifications of individual cortical neurons, triggered by vision and passive eye movement in immobilized kittens. In Pathophysiology of the Visual System: Documenta Ophthalmologica, L. Maffei, ed., Vol. 30, pp. 100-108, Dr. W. Junk, The Hague.

Frégnac, Y., and M. Imbert (1978) Early development of visual cortical cells in normal and dark-reared kittens. Relationship between orientation selectivity and ocular dominance. J. Physiol. (Lond.) 278: 27-44.

Hebb, D. O. (1949) Organization of Behavior, John Wiley and Sons, New York.

Hirsch, H. V. B., and D. N. Spinelli (1970) Visual experience modifies distribution of horizontally and vertically oriented receptive fields in cats. Science 168: 869-871.

Hirsch, H. V. B., and U. N. Spinelli (1971) Modification of the distribution of receptive field orientation in cats by selective visual exposure during development. Exp. Brain Res. 13: 509527.

Hubel, D. H., and T. N. Wiesel (1959) Receptive fields of single neurons in the cat striate cortex. J. Physiol. (Lond.) 148: 574591.

Hubel, D. H., and T. N. Wiesel (1962) Receptive fields, binoc- 
ular interaction and functional architecture in the cat's visual cortex. J. Physiol. (Lond.) 160: 106-154.

Hubel, D. H., and T. N. Wiesel (1963) Receptive fields of cells in striate cortex of very young, visually inexperienced kittens. J. Neurophysiol. 26: 994-1002.

Hubel, D. H., and T. N. Wiesel (1965) Binocular interaction in striate cortex of kittens with artificial squint. J. Neurophysiol. 28: 1041-1059.

Imbert, M., and P. Buisseret (1975) Receptive field characteristics and plastic properties of visual cortical cells in kittens reared with or without visual experience. Exp. Brain Res. 22: $2-36$.

Kasamatsu, T., and J. D. Pettigrew (1976) Depletion of brain catecholamines: Failure of ocular dominance shift after monocular occlusion in kittens. Science 194: 206-209.

Kasamatsu, T., and J. D. Pettigrew (1979) Preservation of binocularity after monocular deprivation in the striate cortex of kittens treated with 6-hydroxydopamine. J. Comp. Neurol. 185: 139-181.

Kohonen, T. (1977) Associative Memory: A System Theoretical Approach, Springer, Berlin.

Kratz, K. E., and P. D. Spear (1976) Effects of visual deprivation and alterations in binocular competition on responses of striate cortex neurons in the cat. J. Comp. Neurol. 170: 141152.

Leventhal, A. G., and H. V. B. Hirsch (1977) Effects of early experience upon orientation selectivity and binocularity of neurons in visual cortex of cats. Proc. Natl. Acad. Sci. U. S. A. 74: $1272-1276$.

Leventhal, A. G., and H. V. B. Hirsch (1980) Receptive field properties of different classes of neurons in visual cortex of normal and dark-reared cats. J. Neurophysiol. 43: 1111-1132.

Marr, D. (1969) A theory of cerebellar cortex. J. Physiol. (Lond.) 202: 437-470.

Movshon, J. A. (1976) Reversal of the physiological effects of monocular deprivation in the kitten's visual cortex. J. Physiol. (Lond.) 261: 125-174.

Nass, M. M., and L. N. Cooper (1975) A theory for the development of feature detecting cells in visual cortex. Biol. $\mathrm{Cy}-$ bern. 19: 1-18.

Perez, R., L. Glass, and R. J. Shlaer (1975) Development of specificity in the cat visual cortex. J. Math. Biol. 1: 275-288.

Pettigrew, J. D. (1978) The paradox of the critical period for striate cortex. In Neuronal Plasticity, C. W. Cotman, ed., pp. 311-330, Raven Press, New York.

Rauschecker, J. P., and W. Singer (1981) The effects of early visual experience on the cat's visual cortex and their possible explanation by Hebb synapses. J. Physiol. (Lond.) 310: 215240.

Sejnowski, T. J. (1977a) Storing covariance with nonlinearly interacting neurons. J. Math. Biol. 4: 303-321.

Sejnowski, T. J. (1977b) Statistical constraints on synaptic plasticity. J. Theor. Biol. 69: 385-389.

Sillito, A. M. (1975) The contribution of inhibitory mechanisms to the receptive field properties of neurons in the striate cortex of the cat. J. Physiol. (Lond.) 250: 305-329.

Singer, W. (1979) Central-core control of visual functions. In Neuroscience Fourth Study Program, F. Schmitt and F. Worden, eds., pp. 1093-1109, MIT Press, Cambridge.

Singer, W. (1980) Central gating of developmental plasticity in the cat striate cortex. Verh. Dtsch. Zool. Ges. 268-274.

Stent, G. S. (1973) A physiological mechanism for Hebb's postulate of learning. Proc. Natl. Acad. Sci. U. S. A. 70: 9971001.

Stryker, M. P., H. Sherk, A. G. Leventhal, and H. V. Hirsch (1978) Physiological consequences for the cat's visual cortex of effectively restricting early visual experience with oriented contours. J. Neurophysiol. 41: 896-909.

Trotter, Y., Y. Frégnac, P. Buisseret (1981) Gating control of developmental plasticity by extraocular proprioception in kitten area 17. In Fourth European Conference on Visual Perception, Gouzieux, France.

Uttley, A. M. (1976) A two pathway theory of conditioning and adaptive pattern recognition. Brain Res. 102: 23-35.

von der Malsburg, C. (1973) Self-organization of orientation sensitive cells in the striate cortex. Kybernetik 14: 85-100.

Watkins, D. W., J. R. Wilson, and S. M. Sherman (1978) Receptive field properties of neurons in binocular and monocular segments of striate cortex in cats raised with binocular lid suture. J. Neurophysiol. 41: 322-337.

Wiesel, T. N., and D. H. Hubel (1963) Single-cell responses in striate cortex of kittens deprived of vision in one eye. $J$. Neurophysiol. 26: 1003-1017.

Wiesel, T. N., and D. H. Hubel (1965) Extent of recovery from the effects of visual deprivation in kittens. J. Neurophysiol. 28: 1060-1072. 